\title{
Knock Tendency Prediction in a High Performance Engine Using LES and Tabulated Chemistry
}

\author{
Stefano Fontanesi, Stefano Paltrinieri, Alessandro D'Adamo and Giuseppe Cantore \\ Univ of Modena and Reggio Emilia \\ Christopher Rutland \\ Univ of Wisconsin Madison
}

\begin{abstract}
The paper reports the application of a look-up table approach within a LES combustion modelling framework for the prediction of knock limit in a highly downsized turbocharged DISI engine.

During experimental investigations at the engine test bed, high cycle-to-cycle variability was detected even for relatively stable peak power / full load operations of the engine, where knock onset severely limited the overall engine performance.

In order to overcome the excessive computational cost of a direct chemical solution within a LES framework, the use of look-up tables for auto-ignition modelling perfectly fits with the strict mesh requirements of a LES simulation, with an acceptable approximation of the actual chemical kinetics.

The model here presented is a totally stand-alone tool for autoignition analysis integrated with look-up table reading from detailed chemical kinetic schemes for gasoline. The look-up table access is provided by a multi-linear interpolating routine internally developed at the "Gruppo Motori (GruMo)" of the University of Modena and Reggio Emilia. As the experimental tests were conducted operating the engine at knock-limited spark advance, the tool is at first validated for three different LES cycles in terms of knock tolerance, i.e. the safety margin to knock occurrence.

As a second stage, the validation of the methodology is performed for discrete spark advance increases in order to assess the sensitivity of the modelling strategy to variations in engine operations. A detailed analysis of the unburnt gas physical state is performed which confirms the knock-limited condition suggested by the experimental tests.
\end{abstract}

CITATION: Fontanesi, S., Paltrinieri, S., D'Adamo, A., Cantore, G. et al., "Knock Tendency Prediction in a High Performance Engine Using LES and Tabulated Chemistry," SAE Int. J. Fuels Lubr. 6(1):2013, doi:10.4271/2013-01-1082.

\section{INTRODUCTION}

In the last few years a return of interest towards gasolinefuelled engines is experienced in the market, as emission concerns (mainly on SOOT and NOx) are complicating the development of "clean" Diesel engines at affordable prices, thus pushing the engine research guidelines into highlysupercharged and downsized spark-ignition (SI) engines. The quest for lower emissions and increased fuel economy has given a new effort in the demand for higher level of thermal efficiency in internal combustion engines (ICEs). To achieve these targets the reduction of the overall engine size and weight are seen as the optimal solution. The commonly accepted practice to obtain this is to highly compress the intake charge by means of turbochargers or superchargers in conjunction with direct injection of fuel within the cylinder to counterbalance the charge heating. In fact, in spark ignition engines increased levels of in-cylinder pressure have always been limited by the arising of abnormal combustion phenomena. In particular, the autoignition of gasoline-like fuel in the outer regions of the combustion chamber prior to the main flame propagation gives rise to a complex and intense system of pressure waves. This phenomenon is commonly named knock. The pressure waves reflect on concrete walls of the combustion chamber, as well as on piston, head and valves, and induce a variety of damaging mechanisms that eventually lead to severe engine failures. These include removal of the lubricant film, increasing friction and wear, and large fluctuations of the heat flux to the combustion chamber walls, affecting the high cycle fatigue strength due to thermo-mechanical loadings.

The use of direct injection of fuel in SI engines (DISI engines) in the recent years has helped in limiting the knock 


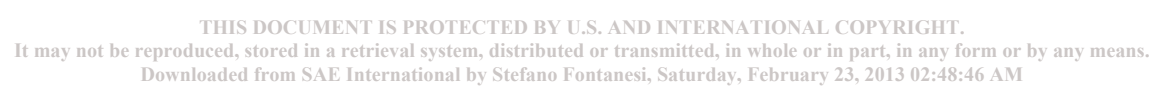

Fontanesi et al / SAE Int. J. Fuels Lubr. / Volume 6, Issue 1(April 2013)

occurrence through the charge cooling effect provided by the evaporation of the fuel inside the combustion chamber. However, with the increasing demand for higher boost pressures, knock still remains one of the most severe performance limiters in SI engines, such as the engine under investigation in the present work. This is made even more complex by cycle-to-cycle variability (CCV) that strongly affects the knock-tendency quality of each single engine cycle. These features are relieved in the investigated engine, a highly-downsized and turbocharged DISI engine currently under production by Ferrari S.p.A.

The engine operating condition investigated in the paper is the peak-power engine speed $(7000 \mathrm{rpm})$ full load one. This high-revving speed, high load and relatively stable operating point, turns out to be severely limited by knock onset, as relieved by experimental measurements carried out by the engine manufacturer. As a consequence, a reduced spark advance is needed in order to limit the probability of abnormal combustion events.

From the point of view of numerical analyses, consolidated CFD simulations based on the ReynoldsAveraged Navier Stokes (RANS) approach are not able to deal with $\mathrm{CCV}$ issues, as the time-average operated in the numeric framework "a priori" removes any fluctuating characteristics of the engine operation. Results from these simulations are ensemble averaged cycles that can be very little useful when dealing with considerable CCV and highly unstable operating conditions such as those very close to knock.

Recent progresses in computational resources made it possible the application of a consolidated numeric approach called Large Eddy Simulation (LES) also to engineering problems and complex geometries such as reciprocating engines [2] [3] . In LES simulations only a filtering in space is carried out, while no filtering in time is performed. The intrinsic unsteadiness of LES modeling of fluids makes it a very well suited tool for CCV analysis of combustion in engines, as it is extensively reported in a previous work [1].

This study extends the aforementioned analysis of CCV by means of LES with a numerical modeling of autoignition, and a complete framework for knocking prediction alongside $\mathrm{CCV}$ is built and validated.

\section{NUMERICAL FRAMEWORK}

\section{Numerical Grid and LES Modeling}

The numerical analyses here reported are part of the dataset presented in [1]. In particular, as the aim of the study is to focus on the combustion process, neither intake or exhaust ports are needed. The initial conditions for each cycle come from the aforementioned dataset of full-cycle analyses, and a solution mapping procedure is performed onto the closed-valve mesh without the ports. The resulting grid is consisting of about 780000 cells at mid-compression stroke and about 236000 cells at Top Dead Center (TDC).
The LES approach chosen for sub-grid term closure is based on the Smagorinsky eddy viscosity model, while for combustion modeling the ECFM3Z-LES is adopted in the framework of Star-CD. Spray modeling and wall heat transfer rely on RANS-derived sub-models and standard wall function, as a full LES modeling is still under development.

The adoption of closed-valve mesh limits the CPU time needed for each calculation to about 24 hours on 24-cores per case, allowing to easily cover several spark advances as well as different engine cycles.

\section{Chemistry Modeling for Gasoline Autoignition}

A complete modelling of actual-fuel combustion is well known to be a task beyond the present limit of combustion chemistry knowledge, as a real fuel is composed by hundreds of different hydrocarbons (branched and straight chain paraffin, aromatics, alcohols etc.). As a consequence, detailed mechanisms for combustion chemistry modelling are available just for binary or tertiary blends, usually called surrogate fuels.

In addition to this, a complete integration of chemistry in CFD codes is usually extremely demanding from a CPU point of view. In fact, every intermediate species invoked by the chemical mechanism has to be accounted for in the CFD analysis, and this introduces an additional transport equation for each of them.

Therefore, reduction strategies for chemical mechanisms have been a strict urgency since a long time. The usual approach adopted is to resume the chemical kinetics of similar hydrocarbons by a unique species ('lumped' or 'skeletal' approaches), or to neglect intermediate reactions and species whose relevance is very low in the overall chemistry description. All these techniques are aimed at reducing the number of species involved in combustion chemistry.

In the past decade much of the modelling effort in fuel chemistry was devoted to Diesel surrogate. This was in line with the research commitment onto Diesel combustion, which in turn highly benefited of these efforts. Gasoline-like fuels were usually represented as a binary blend of isoctane $\left(\mathrm{C}_{8} \mathrm{H}_{18}\right)$ and n-heptane $\left(\mathrm{C}_{7} \mathrm{H}_{16}\right)$. These are commonly referred to as Primary Reference Fuels (PRF). In the recent decades, as engine technology progressed, it became clear that the standard fuel rating based on the RON and MON tests could not accurately represent the fuel behaviour anymore because of the fuel sensitivity (defined as the difference between RON and MON) [4]. From a modelling point of view this introduced the necessity of gasoline surrogate definition as ternary blends, with toluene (Toluene Reference Fuels, TRF) added to the isoctane/n-heptane blend, being it the most important aromatic in real gasoline [5] .

In this study three different blends are evaluated for gasoline surrogate, all of them containing different volume percentages of isoctane and toluene. The toluene presence, 
alongside with the look-up table approach that will be later described, are both intended to include in the CFD analyses the complete autoignition delay characteristics typical of each blend.

In the modelling framework adopted in this work, the combustion chamber is considered as a two-zone region: the former being the region where regular combustion (i.e. deflagration) occurs. Its modelling is on the behalf of a proper combustion model. The second region is relative to the unburnt gas and this is the zone where knocking phenomena can eventually take place.

The numerical analysis of the former is treated by the ECFM-LES combustion model, while for the latter the chemistry progress of the autoignition event is continuously tracked by a dedicated routine that will be later briefly described.

Focusing on the chemistry approach for gasoline autoignition, a chemical mechanism for gasoline surrogate is needed in order to build the autoignition delay database. This is performed once per each fuel model, and all the results (i.e. the autoignition delays) are stored in a tabulated database, which is finally supplied to the CFD code and accessed to during the calculation.

The autoignition delay is defined as the time when the maximum slope for temperature increase is detected. However, parallel testing carried out by the authors with different definitions (e.g. maximum mole fraction of $\mathrm{OH}$ radical) led to very similar results, enforcing the idea that different definitions of delays do not affect the global results.

A multi-linear interpolating routine was developed at Gruppo Motori of University of Modena in order to calculate an approximate delay for each combination of the four variables driving autoignition, i.e. pressure, temperature, mixture strength and residual level. Since the exact cell-wise value is generally not stored as an input point for the database, an exact result for the delay is not available; an interpolating technique amongst the four independent variables is therefore developed. Each variable is bounded within the smallest threshold relieved in the database, then binary interpolation is carried out for two of these, while the third and fourth are kept fixed at the lower-end value. The repetition of this procedure at the fixed higher-end value for the third coordinate gives a first result which is the interpolation of the first three variables. For all these operations, the lower-end value is kept fixed for the fourth one. The repetition of the entire loop for the higher-end fourth variable gives a second point, and the subsequent interpolation between the previously calculated point and this second one is the final result of the interpolation. The procedure is a sequence of linear interpolations, so it is invariant on the choice of the independent variable sequence. To counteract the high non-linearity of chemical kinetics, the physical steps used for the database building are kept as small as possible.

As a final remark, the progress of autoignition chemistry is taken into account by means of a passive transported scalar, whose mass fraction is hereafter called YIG. The interpolated autoignition delay $\tau$ drives the concentration increase of this intermediate species using the correlation by Lafossas et al. []ㅡ:

$$
\frac{d Y I G}{d t}=Y T F \cdot \frac{\sqrt{\tau^{2}+4(1-\tau)^{Y I G} / Y T F}}{\tau}
$$

Autoignition is tracked as soon as the precursor concentration locally equates the fuel tracer concentration YTF. It is then possible to define a Knock Tolerance scalar function, as the local difference between the fuel tracer and the intermediate species $\underline{(2)}$ :

$$
\text { Knock Tolerance }(\vec{x}, t)=\operatorname{YTF}(\vec{x}, t)-Y I G(\vec{x}, t)
$$

This scalar will be used throughout this study as a measure of more or less pronounced knock tendency.

At the present stage of development, this model only allows single-stage autoignition events. However, since the investigated engine operation is a W.O.T. highly charged one with a gasoline-like fuel, double-stage ignition as well as cool flame phenomena are not expected and this approximation is considered acceptable.

Two main advantages characterizing the modelling strategy are here described. First, the adopted framework for autoignition modelling allows the use of results from mechanisms of any level of detail (as in the case of LLNL gasoline surrogate) at a very limited numeric cost, since the CFD code only interpolates the look-up table data for the autoignition delay and it has to solve just one additional transport equation for the intermediate species. No direct chemistry modelling is therefore invoked in the CFD code, thus negligibly affecting the computational demand. Second, the methodology for autoignition prediction is completely passive, so that it can be decoupled from the main combustion event, thus enabling its use in conjunction with any adopted combustion model. Moreover, from a modeling standpoint, this approach constitutes a significant progress with respect to the usual prediction of knock based on the empirical correlations of Douaud \& Eyzat [7]. Even if their efficiency in predicting knock onset phasing has long been recognized and they are still widely adopted, they lack of any chemistry-based fundamental, making their use in this LES approach a undesired approximation.

As a drawback, knock tendency has to be investigated for every cycle because no evidence of knock occurs from pressure fluctuations. Moreover, multi-cycle analyses taking into account the auto-ignition self-enforcing tendency from the previous knocking cycles are not possible at the present stage, and are considered a significant step for methodology improvement. Finally, no post-knock analysis is carried out, as it is considered more significant to trace in detail the proximity to knock rather than exactly evaluate the maximum amplitude of pressure fluctuation and deal with knock metrics. This kind of result is really difficult to correlate with 
the experimental practice, mostly because there is not a standard for knock measurement and every engine manufacturer has its own threshold values based on experience.

\section{Results}

\section{Gasoline surrogate validation}

Three chemical mechanisms and blends have been identified for a first validation of the autoignition modelling:

1. Toluene-nHeptane-Ethanol-Isoctane (hereafter THEO) mechanism by J.C. Andrae et al. []], used in conjunction with a proprietary blend for a commercial RON98 gasoline with no ethanol addition, named RON98-E0 (courtesy of fuel supplier);

2. THEO mechanism by J.C. Andrae et al. [ㅁ] , used for a commercial gasoline with $10 \%$ ethanol content by volume (courtesy of fuel supplier). Even if the octane rating of this blend is slightly lower than RON98, it is named RON98-E10;

3. Lawrence Livermore National Laboratory (LLNL) Gasoline Surrogate Mechanism: a detailed mechanism for gasoline surrogate was recently published by Lawrence Livermore National Laboratory (LLNL) [9]. The surrogate adopted in this study is the one proposed by Vanhove at al [10] consisting of isoctane/toluene/1-hexene (47/35/18 mol. \%).

It worth to point out that both blends based on the THEO mechanisms show a very limited negative temperature coefficient (NTC) characteristic, which is a common feature for blends with a very high content of aromatic (toluene). On the contrary, the LLNL model has a more marked NTC feature. This is expected to result in slightly longer delay times for this fuel blend. Each of the three mechanisms is used to generate a fuel-specific look-up table of autoignition delays by means of a constant pressure reactor model in the framework of DARS-Basic Software [11]. Sample testing of the three mentioned blends in typical engine conditions is reported in Figure 1 and $\underline{2}$.

The $\mathrm{p}, \mathrm{T}, \Phi, \mathrm{EGR}$ ranges are chosen with the intent to fully cover the set of thermo-chemical conditions for unburnt gas relieved in the investigated analysis. The same sampling space is adopted for the three look-up tables, each of them consisting of 25578 analyzed conditions.

A single cycle is chosen amongst the available ones for the preliminary model validation, and three spark advances (SA) are analyzed for the same cycle. The goals pursued for this preliminary validation are:

- absence of knock onset for experimental spark advance, as experiments showed it as the knock limited spark advance condition (KLSA);

- detectable and wide knocking phenomena are expected for a moderate and high increase in SA (respectively $+3 \mathrm{CA}$ and $+6 \mathrm{CA}$ with respect to KLSA).

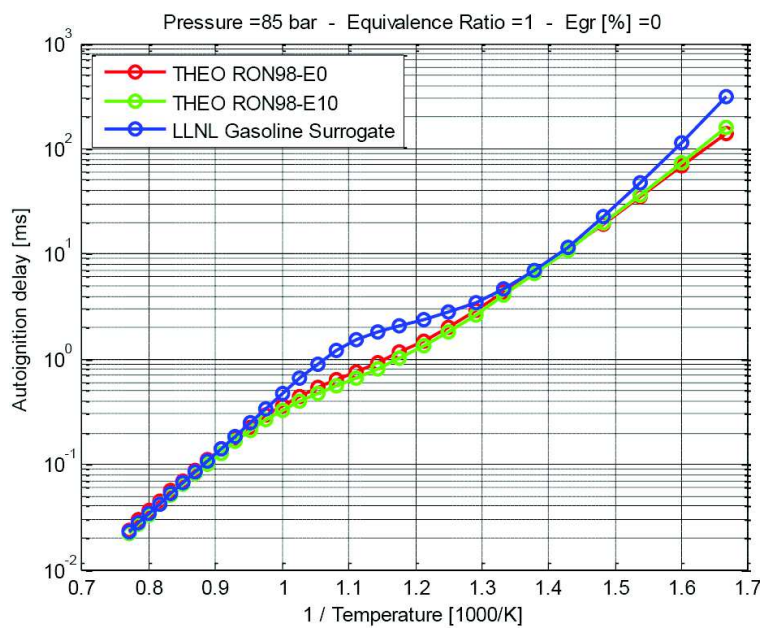

Figure 1. Autoignition delay vs. temperature for stoichiometric mixture at high pressure ( 85 bar), no residuals

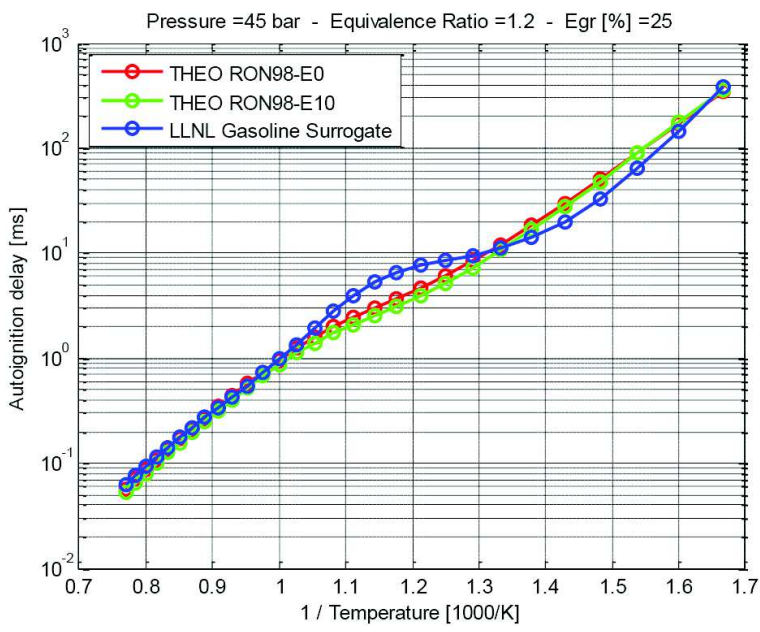

Figure 2. Autoignition delay vs. temperature for rich mixture at intermediate pressure (45 bar), $25 \%$ mole fraction of residuals

Table 1. Summary of Spark Advance investigated to examine proximity to knock onset

\begin{tabular}{|c|c|}
\hline Experimental SA & KLSA \\
\hline Moderate Increase SA & KLSA + 3 \\
\hline High Increase SA & KLSA + 6 \\
\hline
\end{tabular}

The difference arising in combustion progress for the set of examined SA (summarized in Table 1) is clearly visible in Figure 15 in Appendix. 

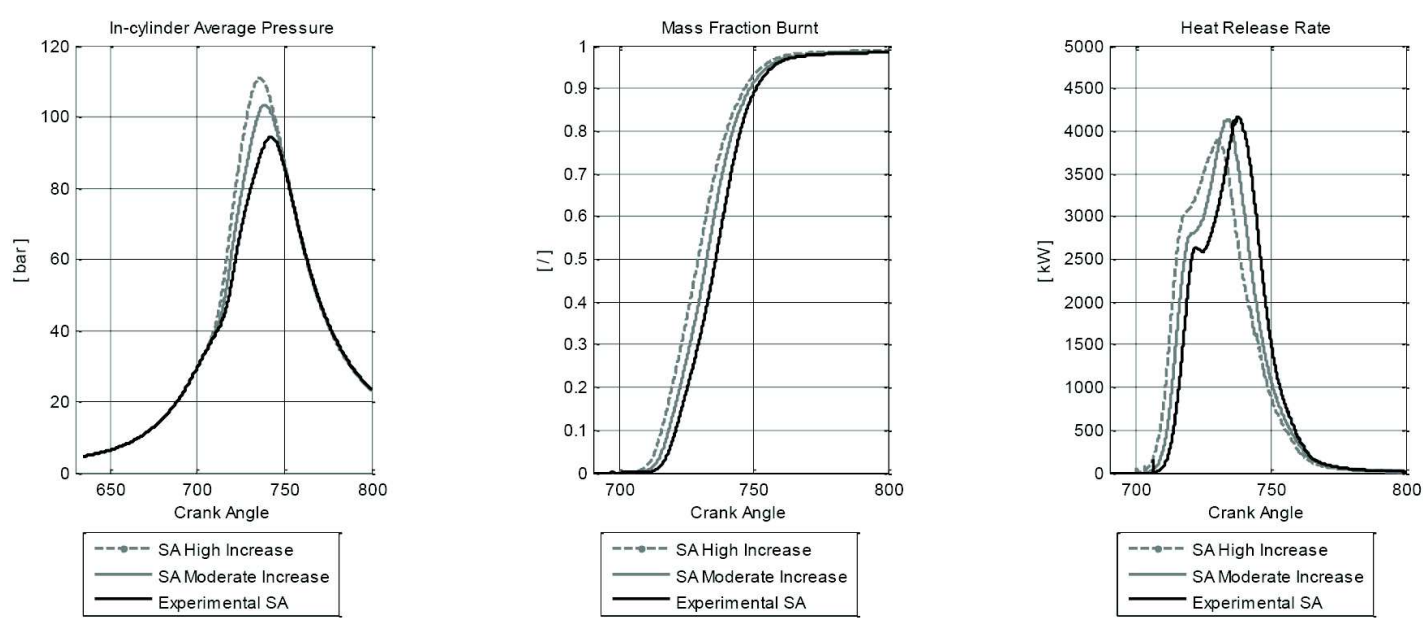

Figure 3. Combustion, Mass Fraction Burnt and Heat Release Rate for the three SA of the same cycle for gasoline surrogate validation

Hereafter the main combustion features of the SA investigated for the same flow realization (i.e. for the same cycle) are reported.

In Figure 3 the main features of combustion are reported to highlight the increased heat release due to advances in SA and the consequent growth in thermodynamic loading for unburnt gas. As previously pointed out, the absence of pressure fluctuations is solely due to the passive knock model adopted and is not an index of regular combustion development. Further investigations are needed in order to assess the absence or not of autoignition events.

\section{Local probe analysis}

A first qualitative analysis of autoignition progress is conducted by means of four local probes localized at the outer border of each valve pocket. The motivation for these measurements is that these regions are well known to be very favourable to knock onset. Therefore, a local tracking of chemistry progress can give particularly useful information on the knock tendency of the operating point. Figure 4 reports the locations assigned for point-wise measurements:

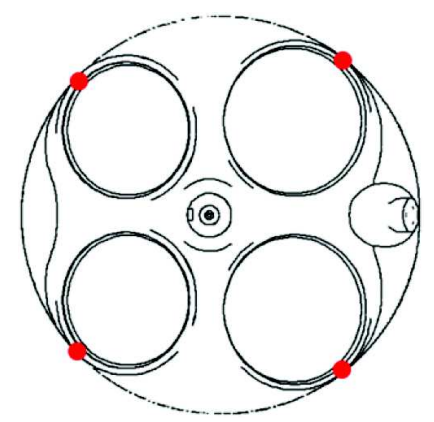

Figure 4. Probes arrangements: intake side on the right of the figure, exhaust side on the left

However, it is important to anticipate that, being local measurements at fixed locations, it is not possible to predict if knock will eventually occur at the very same locations or in a region close to the probes. Thus the presented results are to be intended as purely qualitative and a more detailed analysis will follow in the next sections.

Moreover, this comparison amongst fuel models has to be intended as a general overview on fuel surrogate models: the aim of this section is the assessment of trends common to the three blends that could allow considerations independent of the specific model for gasoline. Therefore, if some of the adopted models predict knocking conditions (or "sufficiently close to knocking" conditions), then particular care should be adopted and the operating point could not be defined as safe from knock in an absolute sense.

In Figures 5a, $, \underline{b}, \underline{c}$ the Knock Tolerance throughout combustion is reported for the three blends for gasoline surrogate, as well as the local combustion progress.

For experimental SA none of the blends under investigation shows zero or negative Knock Tolerance, i.e. verified autoignition criterion. This confirms that in the selected locations the knocking condition is never reached.

For moderate increase in SA, the blends based on the THEO mechanism reach values very close to the critical one (i.e. the null value) for Knock Tolerance.

Finally, for high increase in SA the knocking condition is clearly exceeded for both the THEO-based blends with a local combustion progress still very close to null value, i.e. knocking is verified in a fully unburnt region.

The higher knock tolerance relieved for LLNL Gasoline Surrogate is in agreement with the larger autoignition delays relieved for this blend in the intermediate temperature range, as previously pointed out in Figure 1 and 2 . Considering the high rpm condition investigated, small differences in autoignition delays can results in very different CA for knock onset, and in this case this makes the difference between a verified, intense knock and a knock-safe condition. 

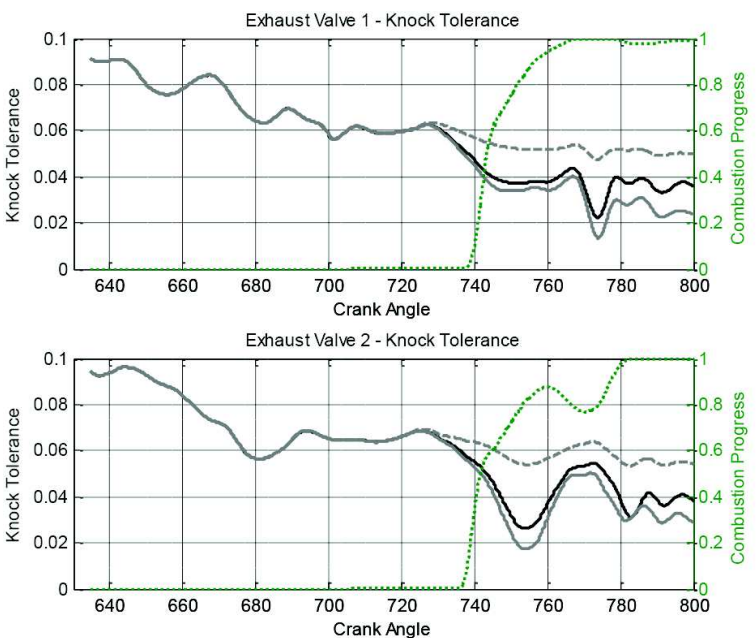
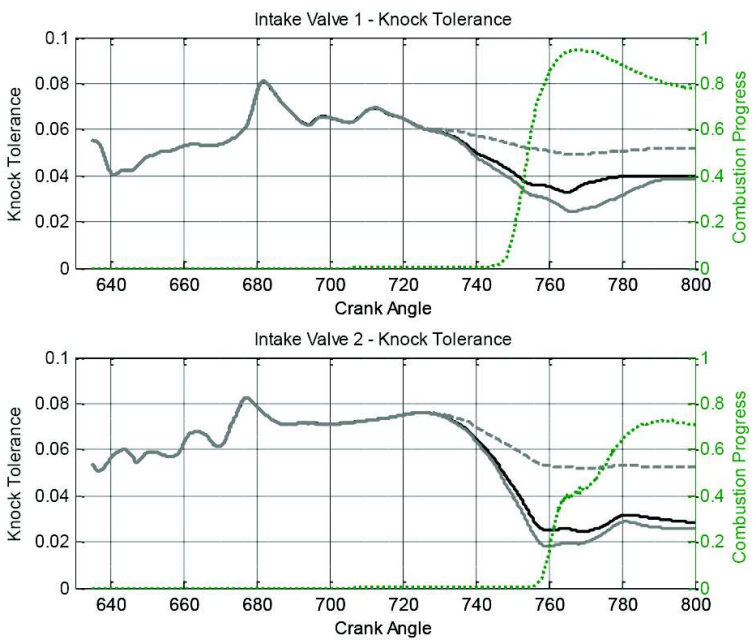

Figure 5a. Probe measurements for Experimental KLSA : Knock Tolerance for RON98-E0 (Black Line), RON98-E10 (Gray Solid Line), LLNL Surrogate (Gray Dashed Line), left scale, and Combustion progress (Green Dotted Line), right scale
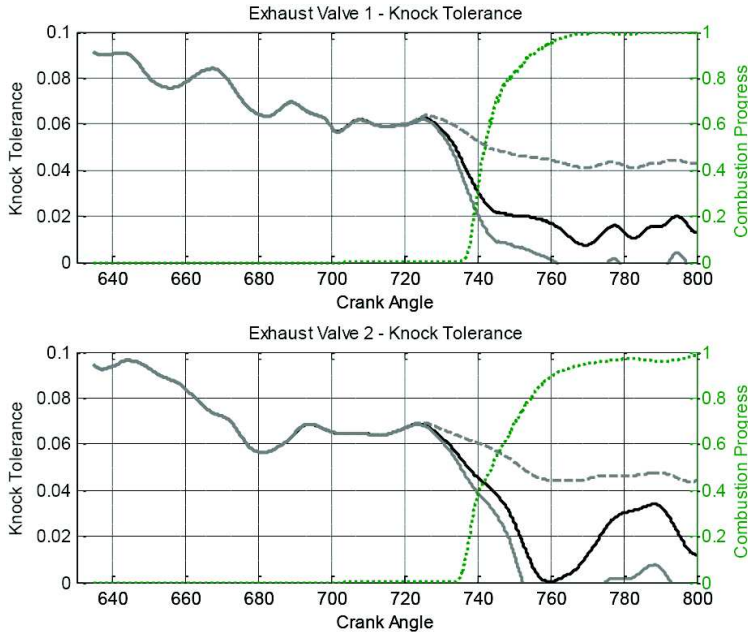
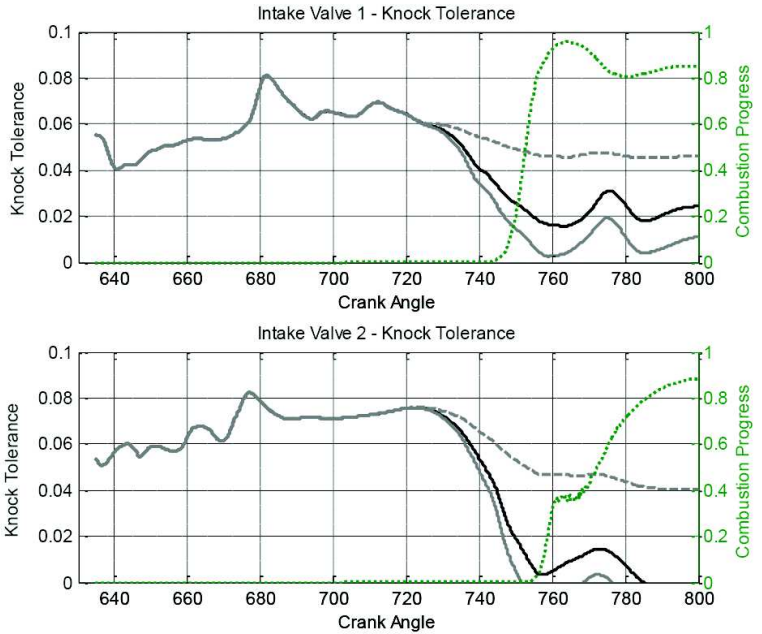

Figure 5b. Probe measurements for moderate increase SA (+3CA from KLSA) : Knock Tolerance for RON98-E0 (Black Line), RON98-E10 (Gray Solid Line), LLNL Surrogate (Gray Dashed Line), left scale, and Combustion progress (Green Dotted Line), right scale

\section{Statistical analysis}

A deeper analysis is then carried out by resuming the unburnt gas state with probability density functions (PDF) for a given $\mathrm{CA}$ position. The combustion chamber is divided into 4 sectors, each one being 90 degrees and including one valve, which gives hereafter its name to the sector. The reference for the subdivision is the geometrical centre of the top-view of the combustion chamber, although the spark plug location is shifted towards the exhaust side of the combustion chamber.

The unburnt gas are identified by mean of a threshold value for progress variable of combustion and they constitute the statistical basis for this analysis.
The result is a set of sector-based PDFs, each of them giving the distribution of the analyzed physical variable for the specific sector at a specific CA.

An immediate and reasonable choice for PDF analysis would be the autoignition delay. The instantaneous PDF of this would give a clear indicator of how fast is the autoignition progress approaching. This measure is closely related to a reaction-rate idea, but gives no indication about the effective proximity of the knock event (i.e. the autoignition chemistry progress status).

On the other side the Knock Tolerance function is more useful as it is the real cumulative function of the autoignition progress and constitutes the comprehensive variable needed. 

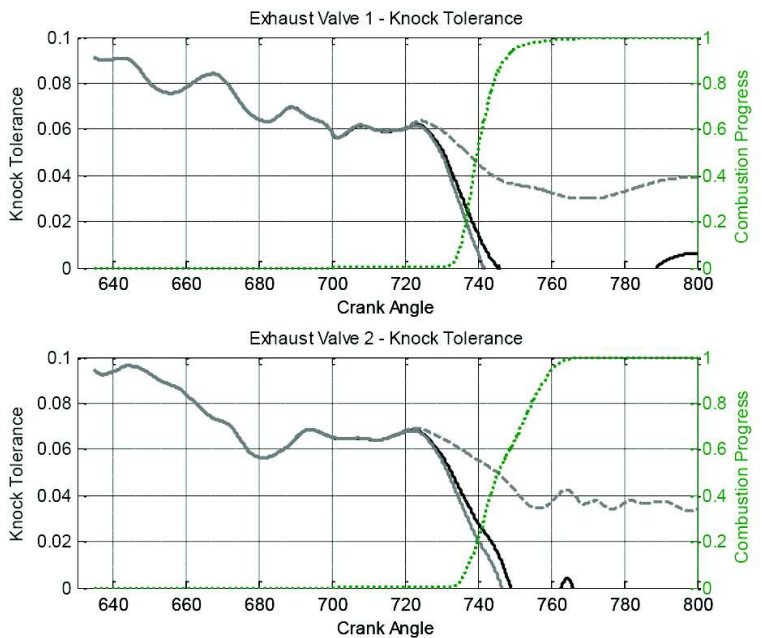
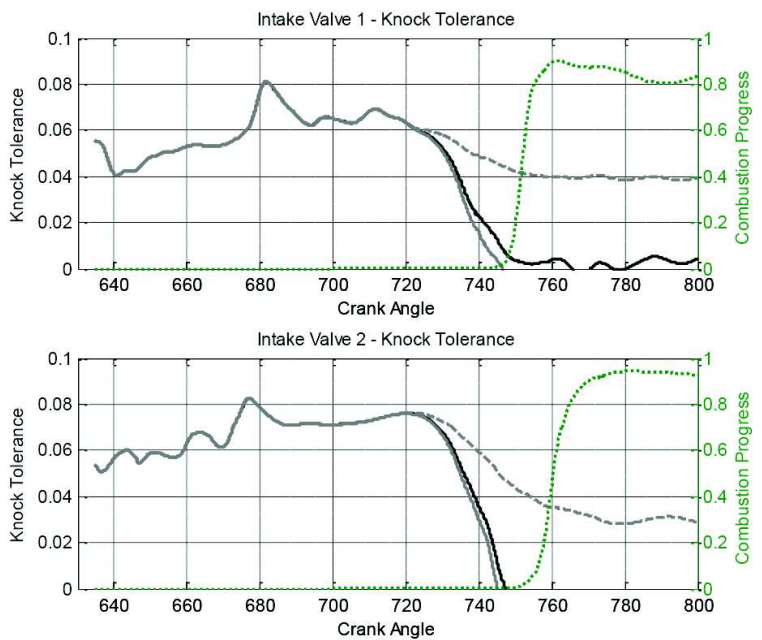

Figure 5c. Probe measurements for high increase SA(+6CA from KLSA): Knock Tolerance for RON98-E0 (Black Line), RON98-E10 (Gray Solid Line), LLNL Surrogate (Gray Dashed Line), left scale, and Combustion progress (Green Dotted Line), right scale
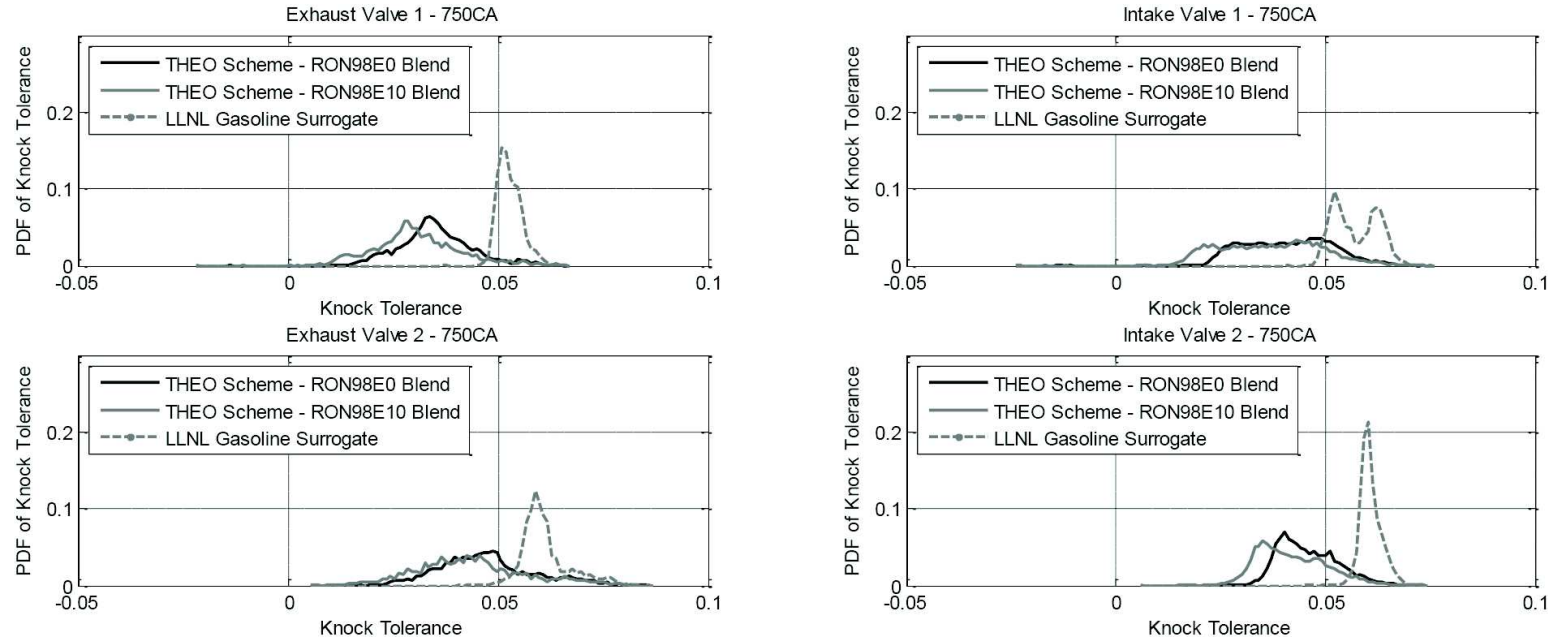

Figure 6a. Gasoline Surrogate Analysis: PDFs of Knock Tolerance for the four sectors of the combustion chamber with Experimental $S A$

Thus when the knock condition is reached and exceeded it will also assume negative values.

For this reason the Knock Tolerance function is chosen as indicator of knock proximity or occurrence.

In order to draw coherent conclusions amongst combustions characterized by different progress histories, due to the progressive increase in SA, a very late crank angle is observed. The observation CA is chosen as 30CA AFTDC, because the mass fraction burnt (MFB) value is very close to $90 \%$ for all the SAs under investigation, as visible in Figure $\underline{3}$. From the PDFs of Knock Tolerance function it is possible to track the proximity of the distribution to autoignition for each of the three analyzed fuel surrogates.
The resulting PDFs for the investigated SA sweeps are reported in Figure $6 a, \underline{b}, \underline{c}$ for the four sectors of the combustion chamber.

Figure 6a refers to the experimental SA and shows that none of the blends is close to the null value for Knock Tolerance at the end of the combustion process. This is a clear indicator that the experimental SA in the simulations never reaches autoigniting conditions for any of the combustion chamber sectors. This result confirms the trend observed in Figure 5a and it is in agreement with experiments.

For a moderate increase in the SA (Figure 6b) nonnegligible probabilities of negative Knock Tolerance are identified for the two blends based on the THEO mechanism. 

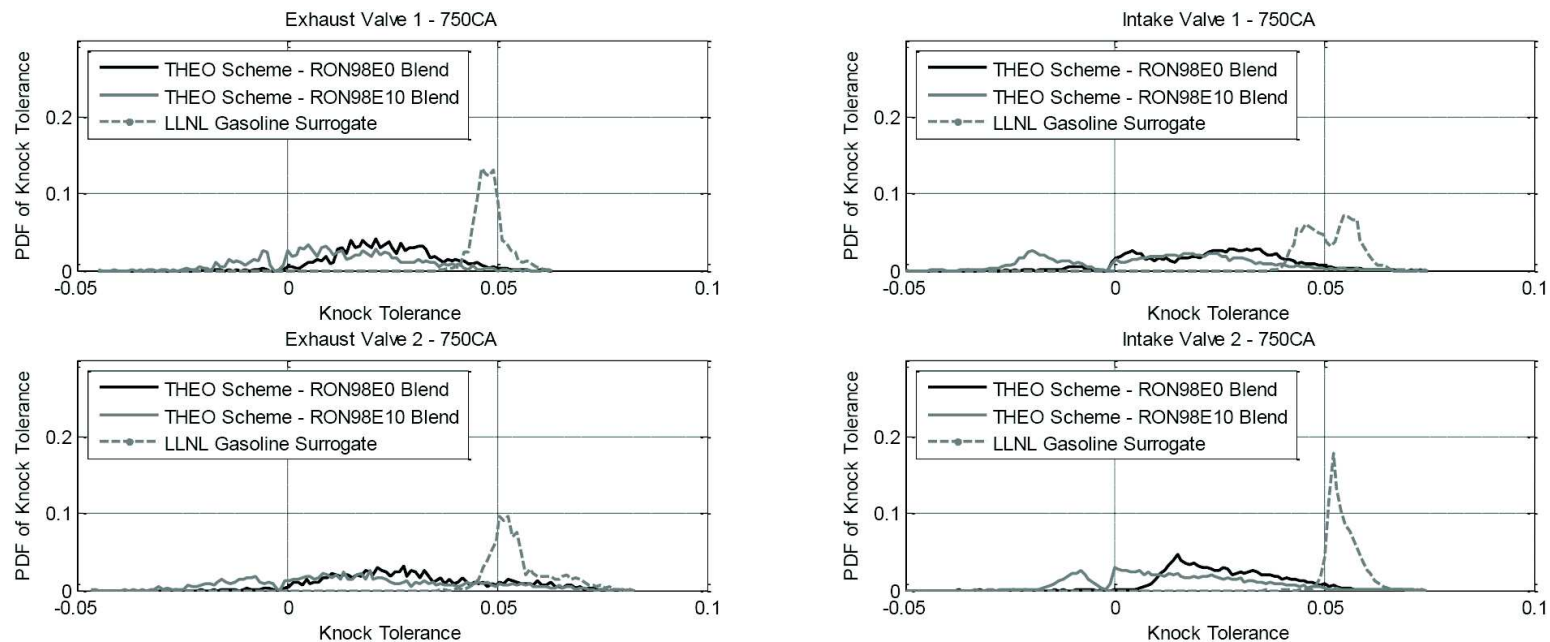

Figure 6b. Gasoline Surrogate Analysis: PDFs of Knock Tolerance for the four sectors of the combustion chamber with Moderate Increase SA
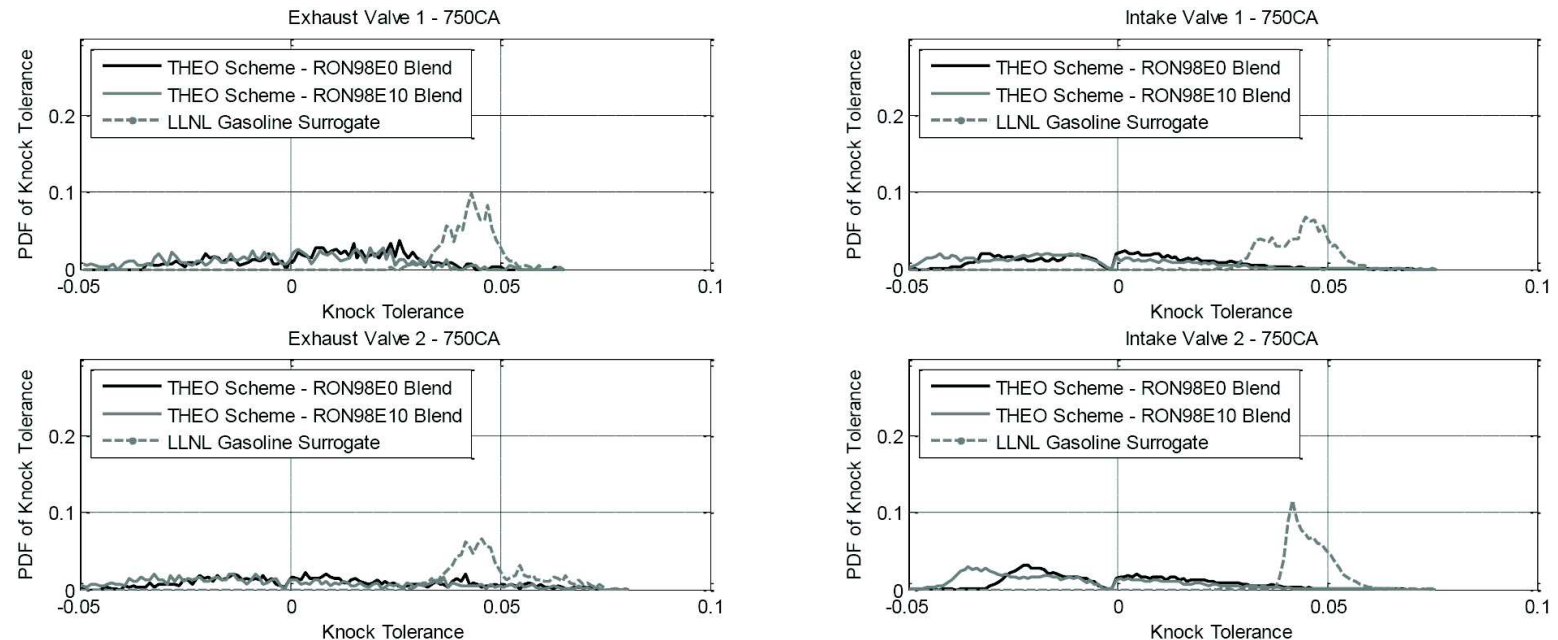

Figure 6c. Gasoline Surrogate Analysis: PDFs of Knock Tolerance for the four sectors of the combustion chamber with High Increase $S A$

Moreover the RON98-E10 blend shows a PDF slightly shifted towards lower values because of its lower octane rating, making this blend more reactive and less resistant to autoignition. The LLNL Gasoline Surrogate still exhibits a non negligible safety margin with respect to autoignition.

In Figure 6c, which is referring to the highly increased SA (+6CA with respect to experimental SA), both blends based on the THEO semidetailed mechanism exhibit approximately half of the samples below the Knock Tolerance null value. Again, this confirms the local observations reported in Figure $\underline{5 c}$ and both indicate a wide and intense autoignition phenomenon. The LLNL Gasoline Surrogate again shows a margin to knocking conditions. However as previously pointed out the goal of this first validation is to assess a general trend that is not relying on a specific blend/ mechanism. Among the three analyzed SAs, only the experimental one achieves safety conditions with all the gasoline surrogates used for autoignition modeling. For a moderate increase of SA some of the chemistry schemes show an onset of autoignition, and an even more critical situation is observed for a higher increase in SA.

As the THEO-base blends were modelled by the fuel supplier of the investigated engine, they are considered as the reference ones for this work.

Moreover, differences can be seen between the different chemical schemes and blends used for the analysis. These are expected, due to the different level of detail of the mechanisms (138 species for THEO and 1389 for LLNL Gasoline Surrogate). These differences amongst the chemical 

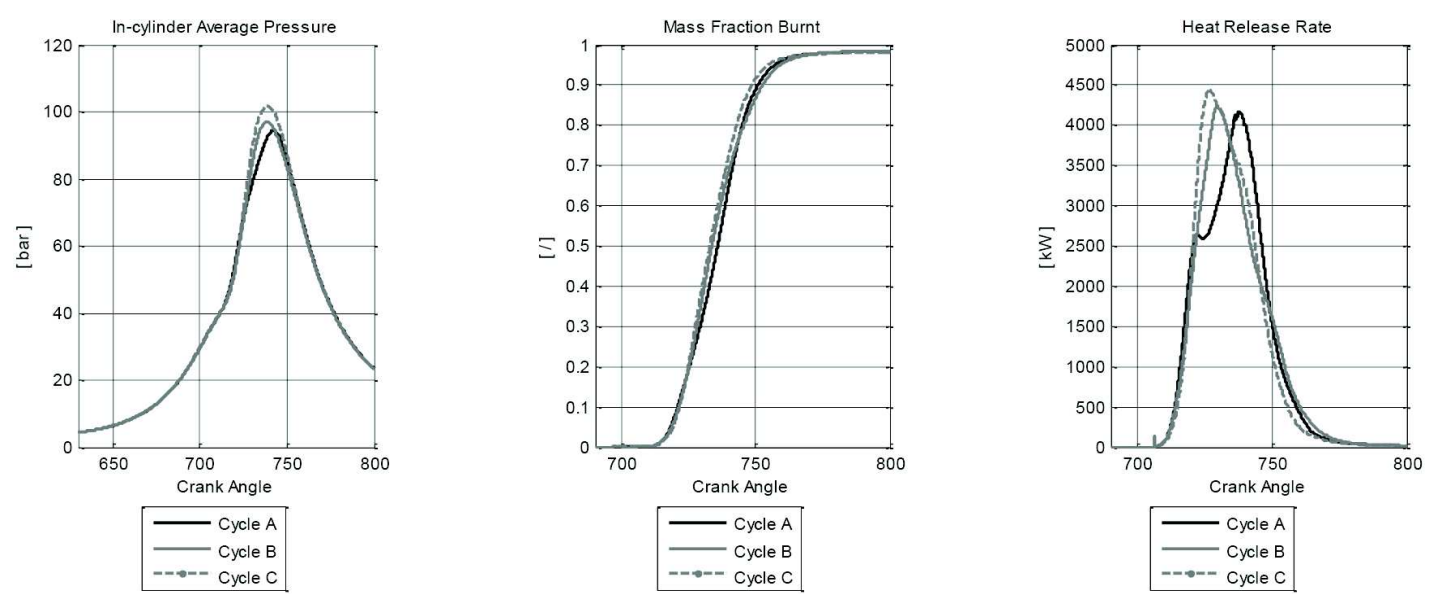

Figure 7a. In-cylinder pressure, fraction of burnt fuel and heat release rate for the three most extreme cycles with experimental $\boldsymbol{S A}$

models are considered acceptable to validate a methodology that is not fuel-dependent.

The remarkable result is that for none of the three mechanisms/blends a PDF for negative values of the Knock Tolerance function occurs for the experimental SA, and this is in agreement with the experimental operating point.

For a moderate increase in SA, there is a non-negligible probability to measure negative values of the Knock Tolerance function. This means that there are fluid cells where the numeric criterion for autoignition is met and exceeded.

It is very important to remember that the cycle under investigation is a sample one and $\mathrm{CCV}$ will have to be considered. This pushes the urgency to discard this moderately increased SA, as even a single cycle analysis does not completely satisfies the knock-safe condition requirement.

The presented set of results confirms the knock-limited condition for the experimental SA and therefore constitutes a validation on the predictive capability of the tool here presented.

A further result from the SA sweep comparison is that when the autoigniting condition occurs, the two sides of the combustion chamber (i.e. intake side and exhaust side) are comparable in terms of knock proximity. This is clearly visible by a visual comparison of the shapes and the position of the PDFs reported in Figure $6 \mathrm{a}, \underline{6 \mathrm{~b}}$ and $\underline{6 \mathrm{c}}$.

This result is noticeable if considering the higher heat flux coming from the exhaust valves; therefore an open issue rises about the knock tendency of the intake side of the combustion chamber. It is worthwhile to underline that the PDF is an intensive representation of the unburnt physical state, i.e. it does not give any information on the mass of unburnt gas. A more complete comparison of the two sides of the combustion chamber will be drawn in the next section.

\section{Effects of CCV on knock}

As the tool for AI prediction is validated against experimental conditions for a single cycle with different chemical schemes for gasoline chemistry, the following analyses deal with the application of the proposed tool to different combustion cycles. The aim of this second part of the study is therefore to verify whether the amplitude of cycle-to-cycle variations (CCV) predicted by the LES simulations is still in agreement with the experimental evidence of KLSA condition or not. The use of this dataset of LES simulations is justified by a previous validation on CCV levels performed by Fontanesi et al. [1] , thus this constitutes a valuable benchmark.

The approach adopted hereafter can be considered complementary to that of the first set of results: a single chemical blend is now chosen for the gasoline chemistry (THEO scheme, with RON98-E0 blend as suggested best fuel model from the fuel supplier) and three different cycles (i.e. different flow realizations) are analyzed operating the engine under the same SA pattern as before (experimental SA, +3CA and $+6 \mathrm{CA}$ with respect to experimental one).

The three cycles are chosen with the specific aim to test the autoignition tool under the broadest range of conditions available within the LES dataset. For this purpose the most extreme cycles available are selected and are hereafter named Cycle A, Cycle B and Cycle C. Cycle A is the cycle identified for gasoline surrogate comparison in the previous section.

Visualizations of combustion development for the three SA are provided in Appendix (Figures 16, 17 and 18), as well as knock onset locations.

\section{Local probe analysis}

The preliminary analysis by means of the same set of four local probes is repeated on each of the three cycles. Albeit these results are once again affected by the choice of an arbitrary sensor position, they prove their efficiency in giving 

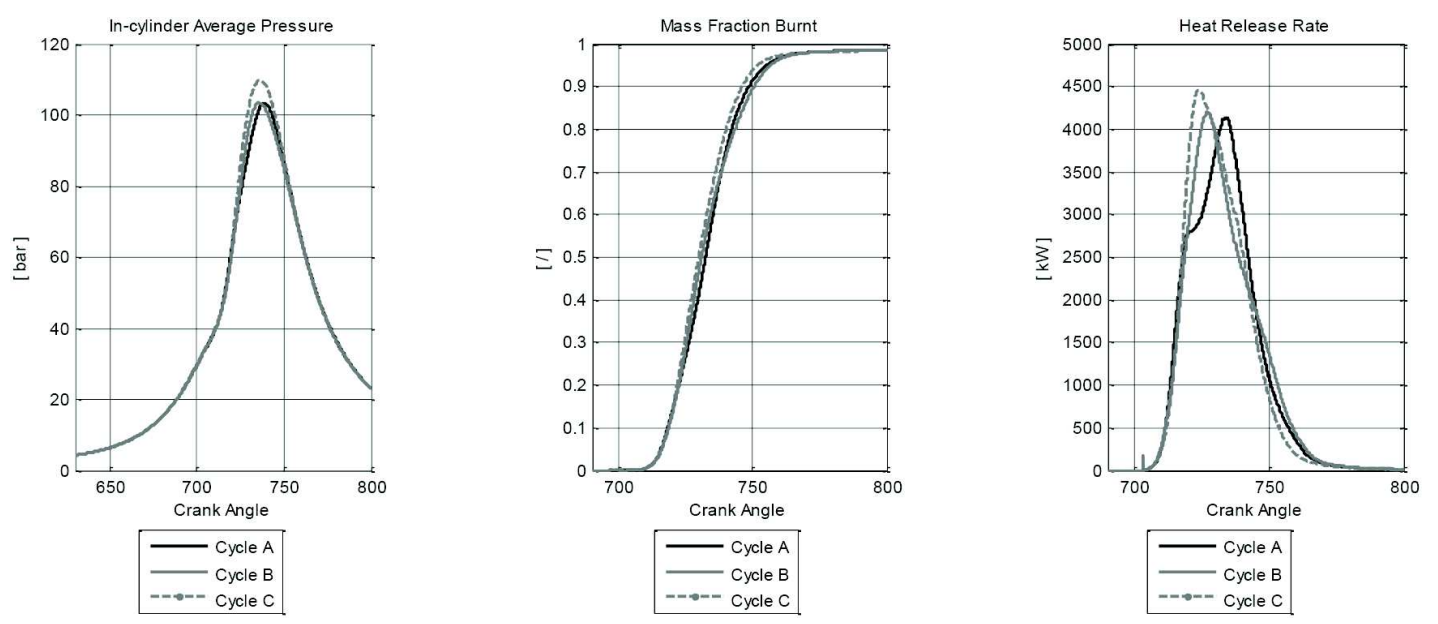

Figure 7b. In-cylinder pressure, fraction of burnt fuel and heat release rate for the three most extreme cycles with moderate increase $S A$
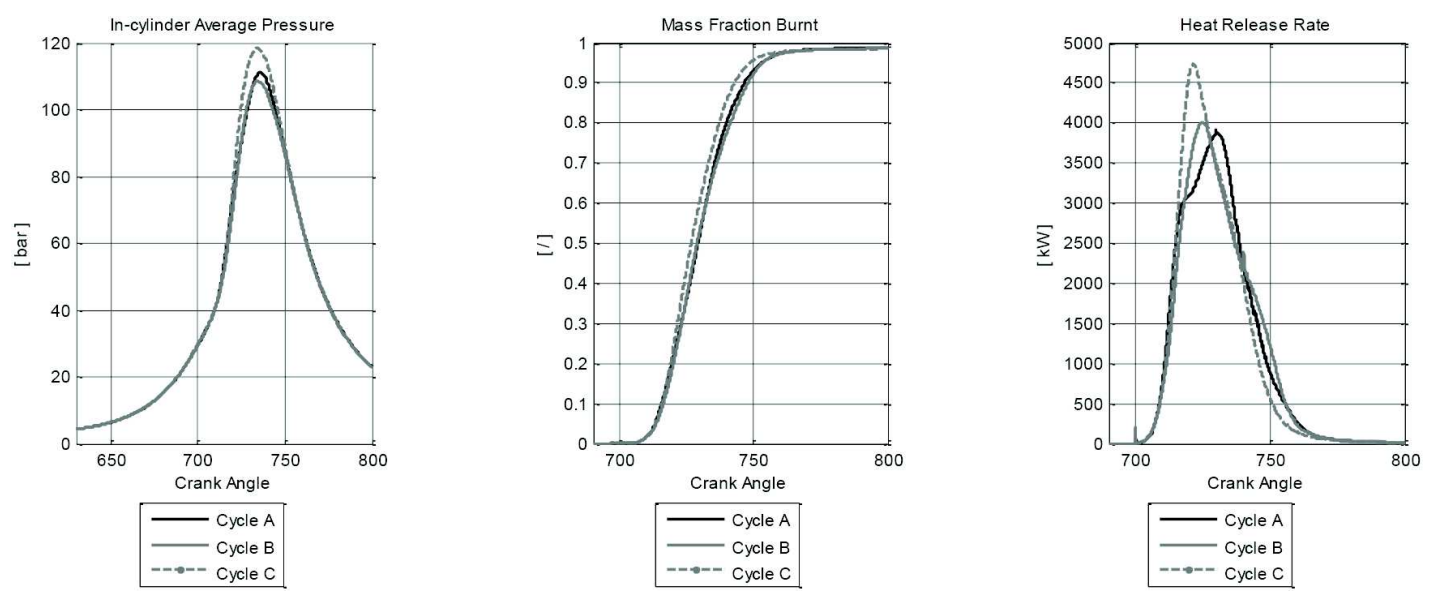

Figure 7c. In-cylinder pressure, fraction of burnt fuel and heat release rate for the three most extreme cycles with high increase $S \boldsymbol{A}$

a first insight into the autoignition characteristics at very knock-susceptible locations.

Under the experimental SA, autoignition conditions are reached in a very limited set of engine locations and for very late CAs, i.e. when the main combustion event is almost completed. These results can be explained considering that knock conditions can be verified within the thin layer of unburnt gas adjacent to the cold walls, where the flame front is extinguishing. Despite the fact that local fresh charge pockets can eventually reach point-wise autoignition conditions, no knock is expected to occur because of both the very limited mass of fresh charge involved in the process and the presence of fully burnt gases surrounding the fresh charge spots. For any SA increase a clear trend emerges, with the local Knock Tolerance abruptly decreasing to null values. Once again, to completely characterize the unburnt gas physical and chemical state, a statistical description is carried out in the next section.

\section{Statistical analysis}

The same procedure previously outlined for the statistical description of the unburnt gases in terms of autoignition proximity is here applied to the three analyzed LES cycles. The application consists therefore of a matrix of three different engine cycles (chosen with the aim of capturing the highest level of CCV within the available LES database) and three different SAs. The observations are all relative to $+30 \mathrm{CA}$ ATDC, being the burnt mass fraction close to $90 \%$ for all the cases, as previously discussed.

A necessary enhancement from the previously described statistical analysis is a filtering procedure on unburnt gas. This is first carried out in order to operate statistical analysis on a coherent dataset. As different cycles (i.e. flow realizations) are examined, each one with its proper 

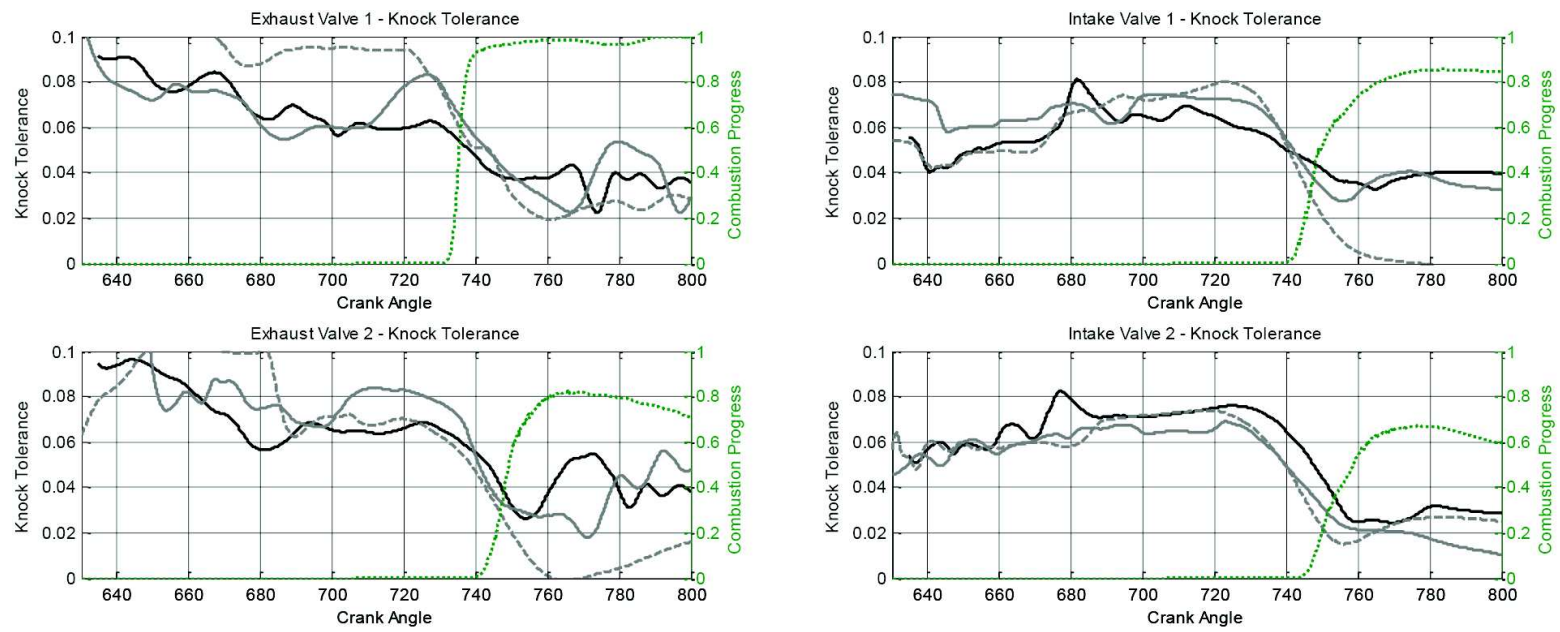

Figure 8a. Knock Tolerance and Combustion progress for the four local probes under Experimental SA (Cycle A black, Cycle B - gray solid, Cycle C - gray dashed)
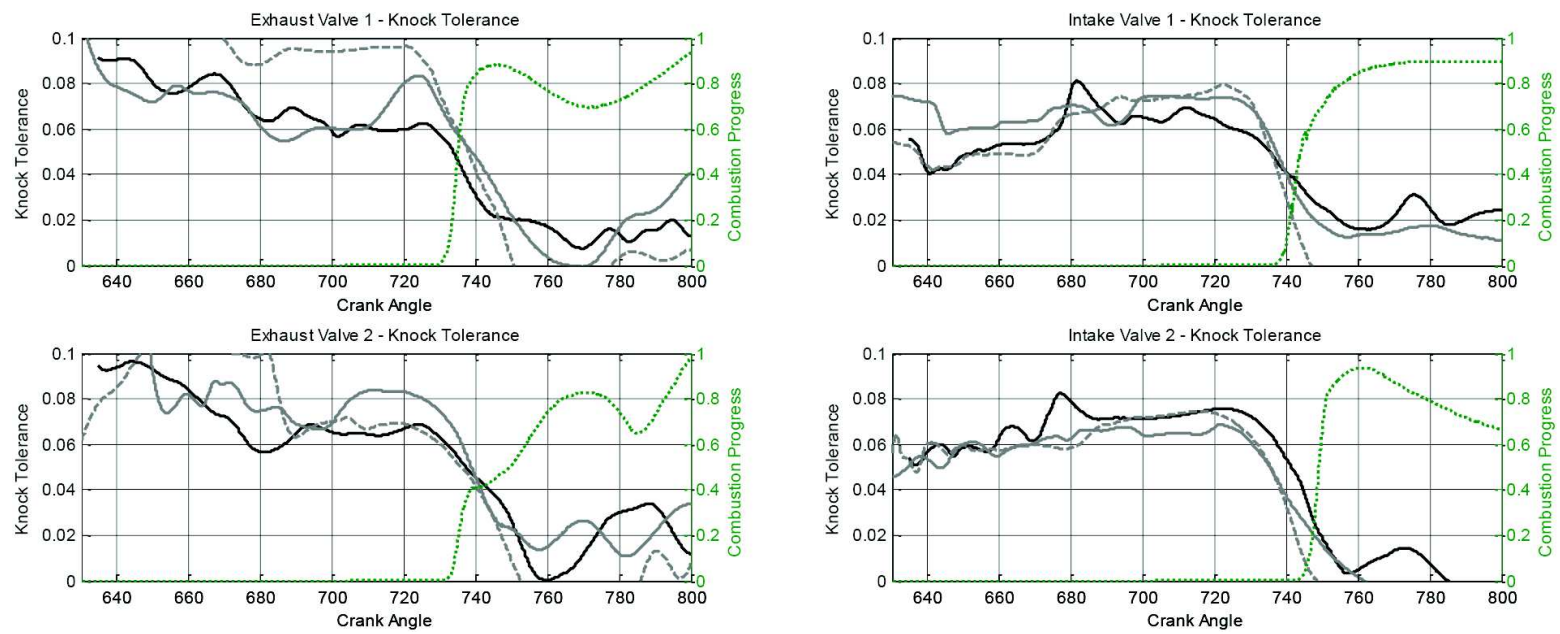

Figure 8b. Knock Tolerance and Combustion progress for the four local probes under Moderate Increase SA (Cycle A - black, Cycle B - gray solid, Cycle C - gray dashed)

combustion development, the volume of unburnt gas may vary significantly from one cycle to another. This would make the statistical analysis volume-dependent, while a more intensive study of the physical state of the unburnt gas is pursued in this stage.

For this reason, only the fluid cells that for a given CA stay below a threshold value of the progress variable in all the combustion cycles investigated are considered as 'unburnt'. The underlying idea is that those cells being above the threshold value for just some of the realizations, they are subjected to the flame brush variability amongst different cycles. Therefore, they cannot be properly addressed to as 'end gas' and they can be discarded from the dataset for knock analysis without any significant loss in the statistical collection. The threshold value for unburnt filtering is kept the same as the previous section.
A first visualization of the proximity to knocking conditions in the current set of LES simulation is given by scatter plots of autoignition-related variables for unburnt gas. As previously described, the knock model relies on the comparison between two passive species: the intermediate precursor for autoignition YIG and the fuel tracer YTF. These can be used as graphic coordinates for a visual description of knock proximity. Each unburnt fluid cell is represented by a point, whose autoignition chemistry progress can be tracked by the precursor species. During combustion, the point cloud progressively approaches the knock limit line, i.e. the $\mathrm{YIG}=\mathrm{YTF}$ condition where knock occurs.

Depending on the combustion history, which is specific for each combination of LES cycle and SA, the knock limit line is reached and eventually crossed at different CAs and by different populations of computational cells. 

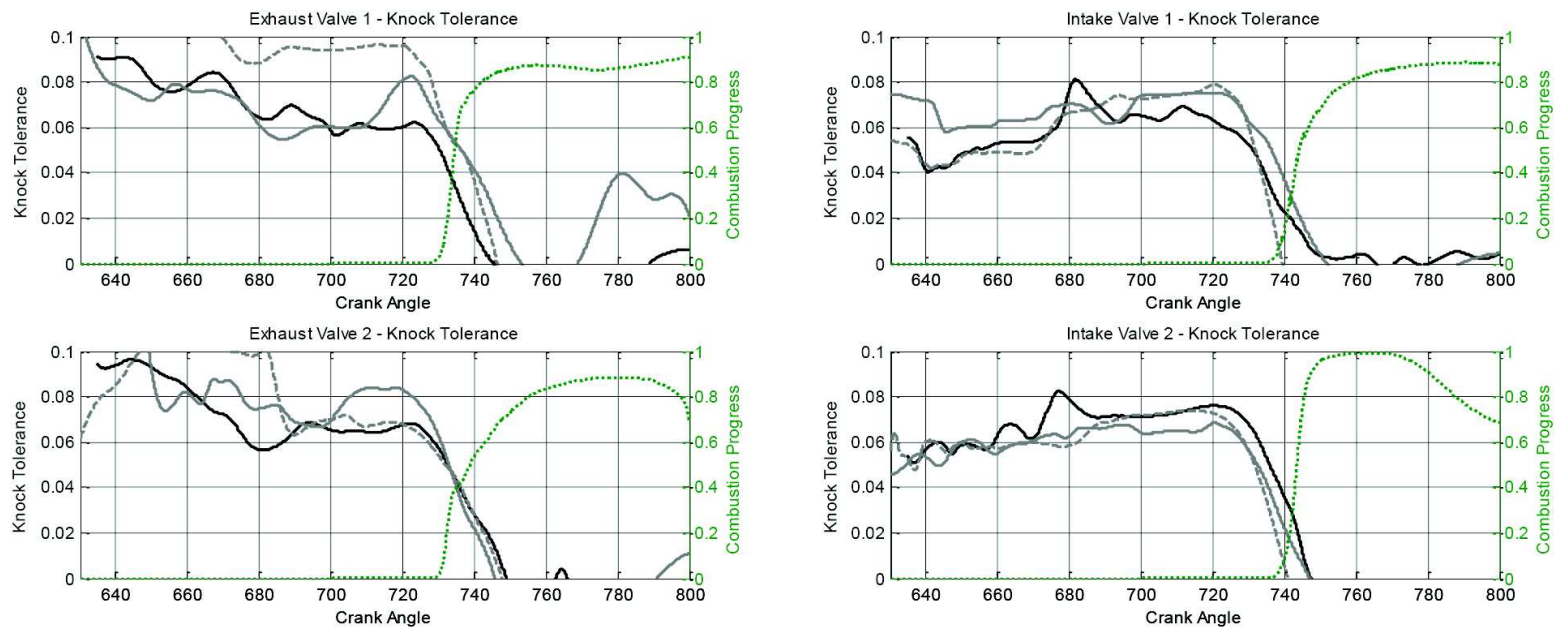

Figure 8c. Knock Tolerance and Combustion progress for the four local probes under High Increase SA (Cycle A - black, Cycle $B$ - gray solid, Cycle C - gray dashed)
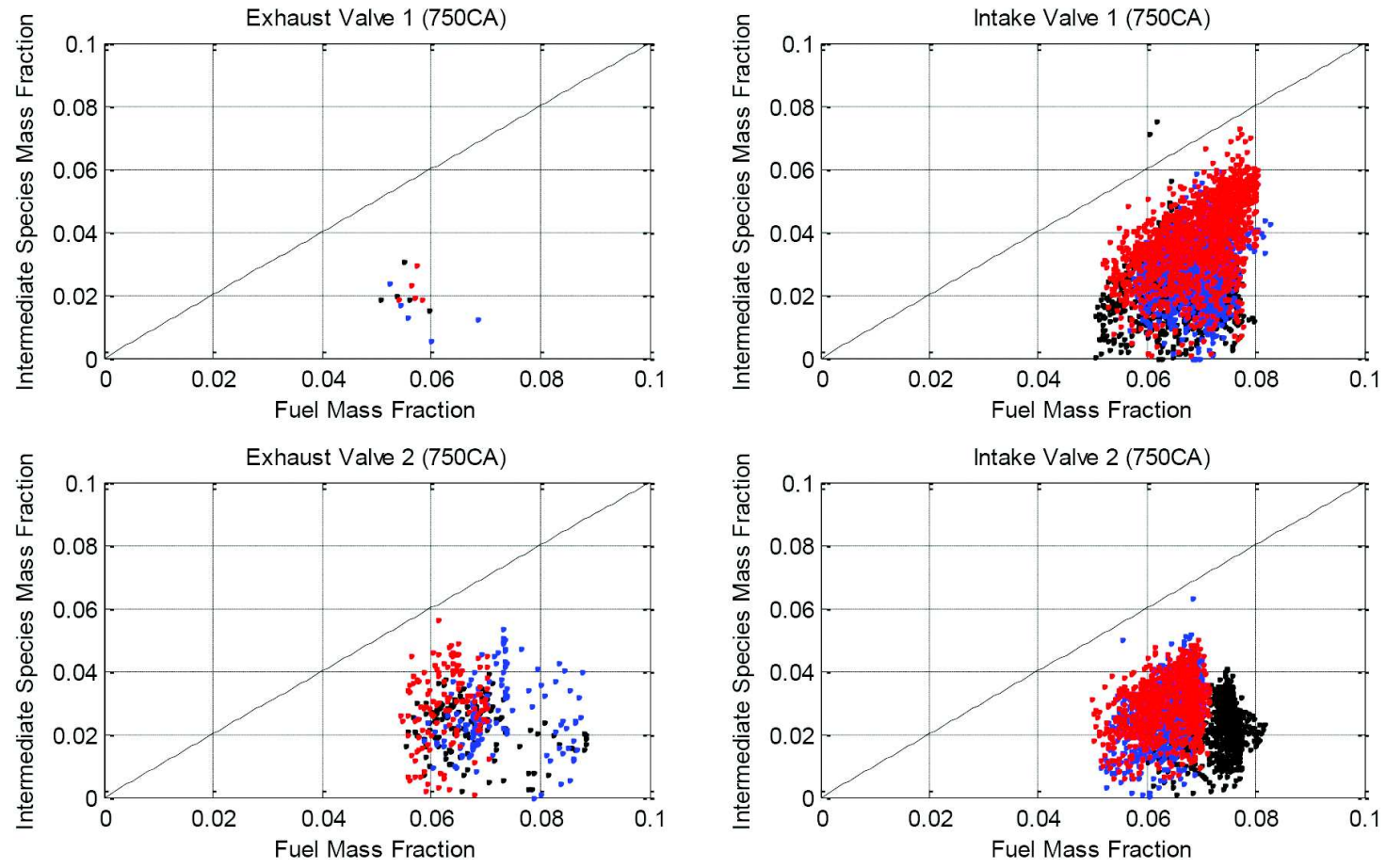

Figure 9a. CCV Analysis : Scatter Plot of Knock Tolerance for the four sectors of the combustion chamber with Experimental SA (Cycle A - black, Cycle B - blue, Cycle C-red)

From the above plots valuable considerations can be drawn. A first observation allows to confirm that for the experimental SA (Figure 9a) the knock limit line is never reached for each of the three cycles. As previously anticipated, these are chosen in order to introduce the maximum level of CCV compatible with experiments, with the aim to broadly test the autoignition analysis tool. The results confirm that no autoignition is predicted operating the engine under the experimental SA, in good agreement with the operating KLSA condition. Even for a moderate increase in SA (Figure 9b) a relevant population of points (i.e. unburnt cells) crosses the knock limit line, so that autoignition can be expected at least for some cycles within the CCV range. The situation is even worse with a further increase in SA (Figure 9c), with all the cycles exhibiting autoignition conditions. Figure $9 \mathrm{~b}$ and $9 \mathrm{c}$ relative to increases in SA indicate that the range of thermodynamic conditions inherent to the predicted level of CCV eventually falls over the autoignition limits of 

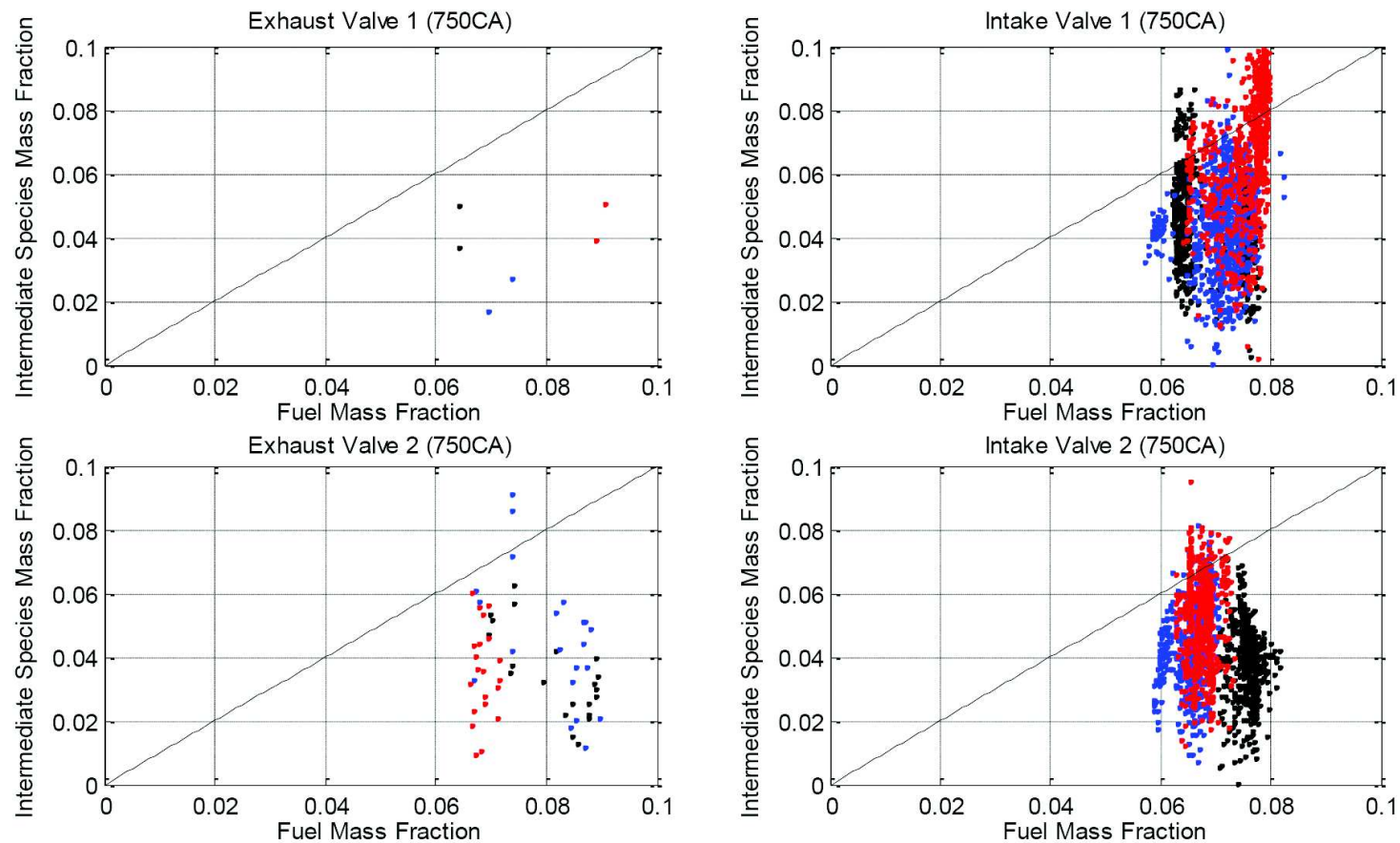

Figure 9b. CCV Analysis : Scatter Plot of Knock Tolerance for the four sectors of the combustion chamber with Moderate Increase SA (Cycle A - black, Cycle B - blue, Cycle C - red)
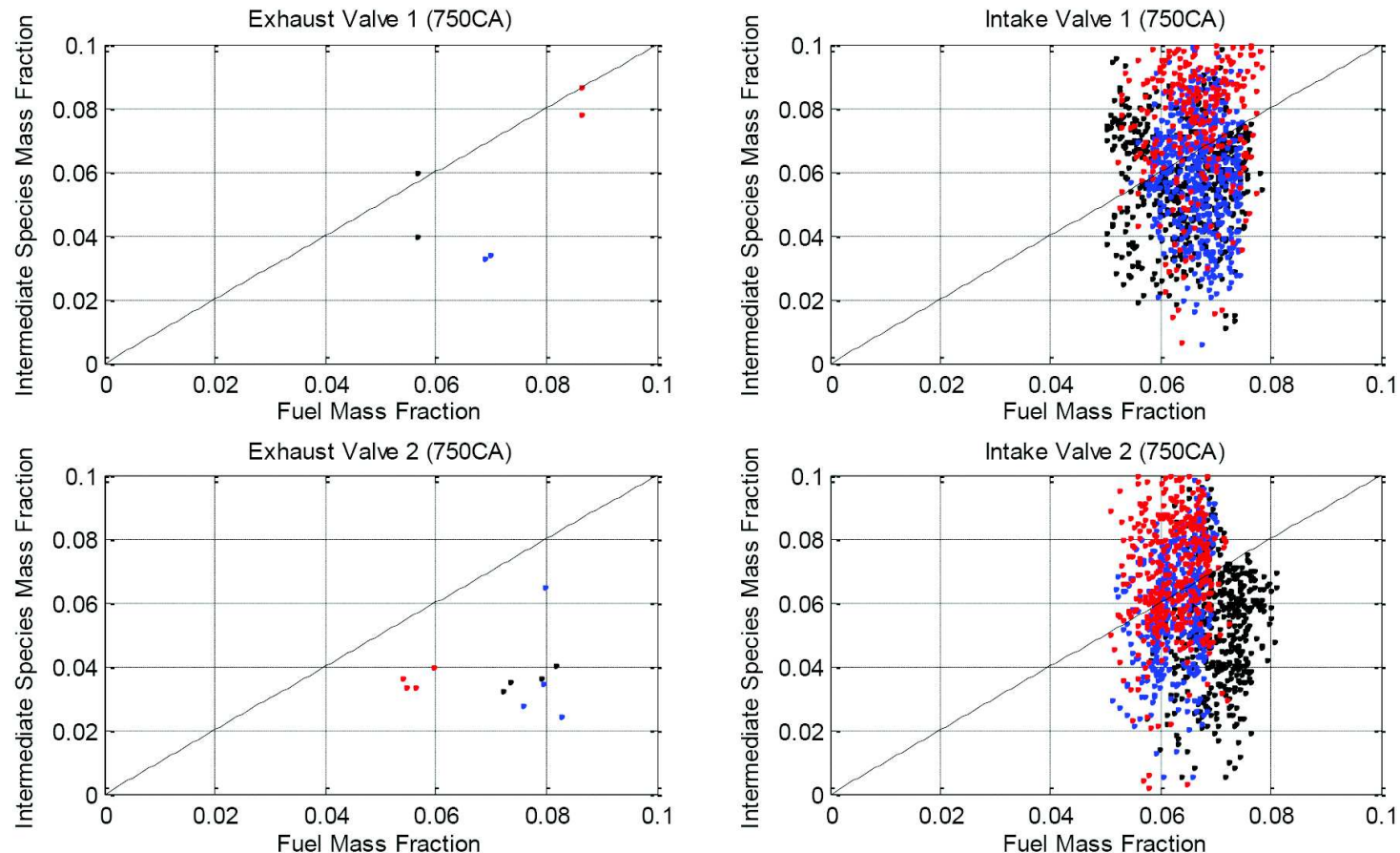

Figure 9c. CCV Analysis : Scatter Plot of Knock Tolerance for the four sectors of the combustion chamber with High Increase SA (Cycle A - black, Cycle B - blue, Cycle C - red) 
the fresh charge, thus knock events are to be expected over a wide set of engine cycles. A second observation from Figures $\underline{9 \mathrm{a}}, \underline{9 \mathrm{~b}}$ and $\underline{9 \mathrm{c}}$ is that for the same CA, the number of unburnt cells is extremely lower in the exhaust side than in the intake one. This is a clear indicator of an unbalanced flame displacement towards the exhaust side and this will be further investigated in the last section.

Concerning the unburnt charge state, the attention can be focused on the intake side of the combustion chamber, since it appears to be more representative of the autoignition progress dynamics. The previous scatter plots can be represented in the form of PDF distribution of the Knock Tolerance function.

In Figures 10a, $\underline{\mathrm{b}}, \underline{\mathrm{c}}$ the trend of knock tendency given by the PDFs reflects the overall in-cylinder pressure depicted in Figures $7 \mathrm{a}, \underline{\mathrm{b}}, \underline{\mathrm{c}}$, with Cycle $\mathrm{C}$ characterized by higher pressure level and lower Knock Tolerance values than Cycle A and Cycle B.

Figure 10a confirms the evaluations drawn from the scatter plots presented in Figure 9a, i.e. none of the investigated cycles showing a PDF of Knock Tolerance function close to zero. Again, increased knock tendency in seen for moderate as well as high increases in SA (Figure 10b and $\underline{10 \mathrm{c}}$ ).
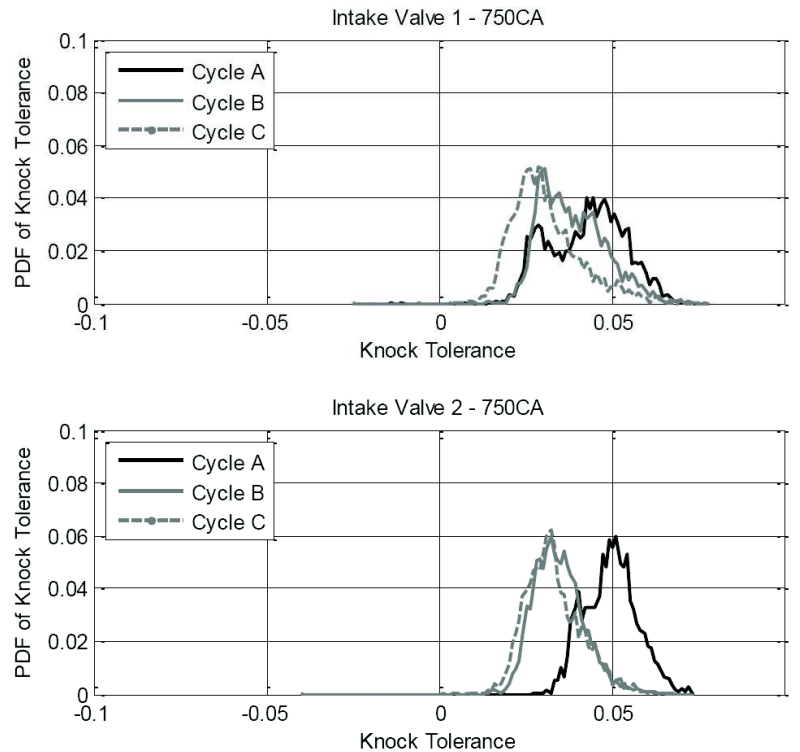

Figure 10a. CCV Analysis : PDFs of Knock Tolerance for the intake side of the combustion chamber with Experimental $S A$
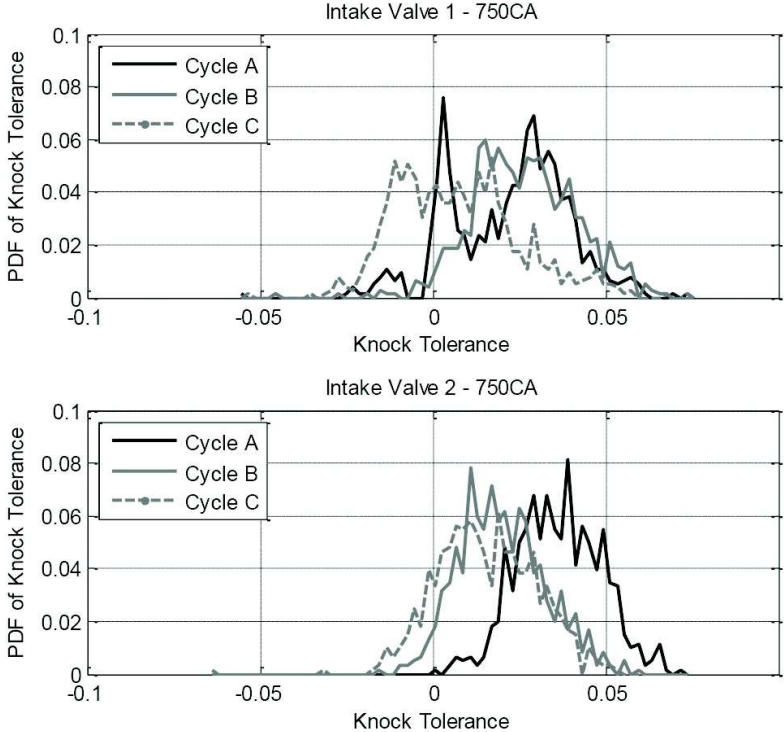

Figure 10b. CCV Analysis : PDFs of Knock Tolerance for the intake side of the combustion chamber with Moderate Increase $\boldsymbol{S A}$
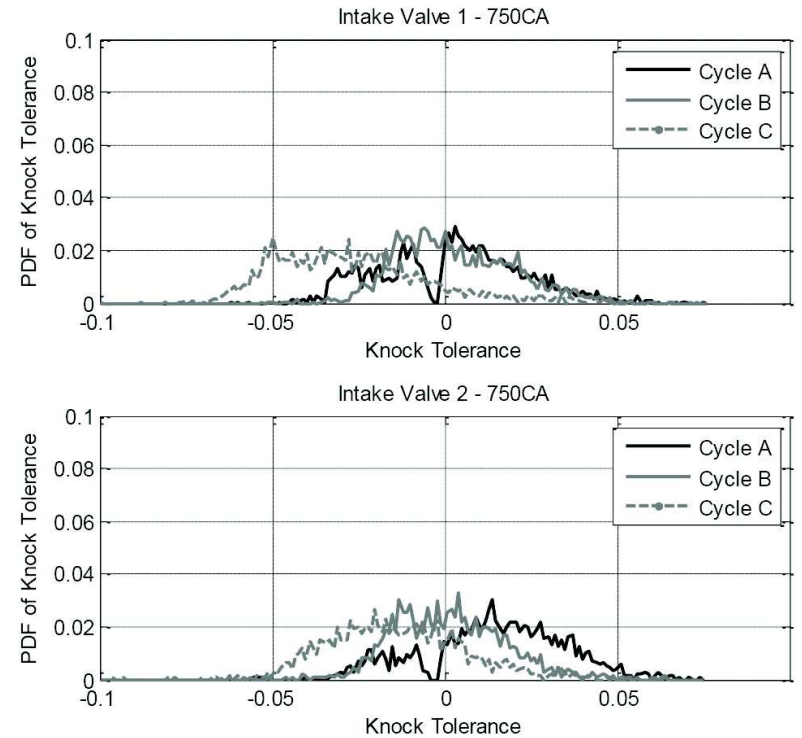

Figure 10c. CCV Analysis : PDFs of Knock Tolerance for the intake side of the combustion chamber with High Increase $S A$

\section{Combustion development analysis}

The final part of the study is devoted to analyze how the main flame front propagates inside the combustion chamber. In particular, a critical analysis is carried out regarding whether the flame's main features are optimized with regard 

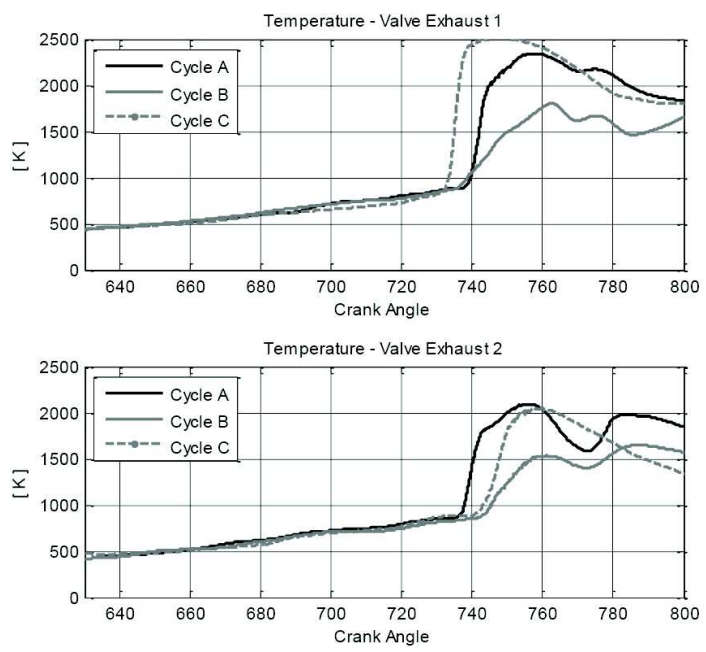
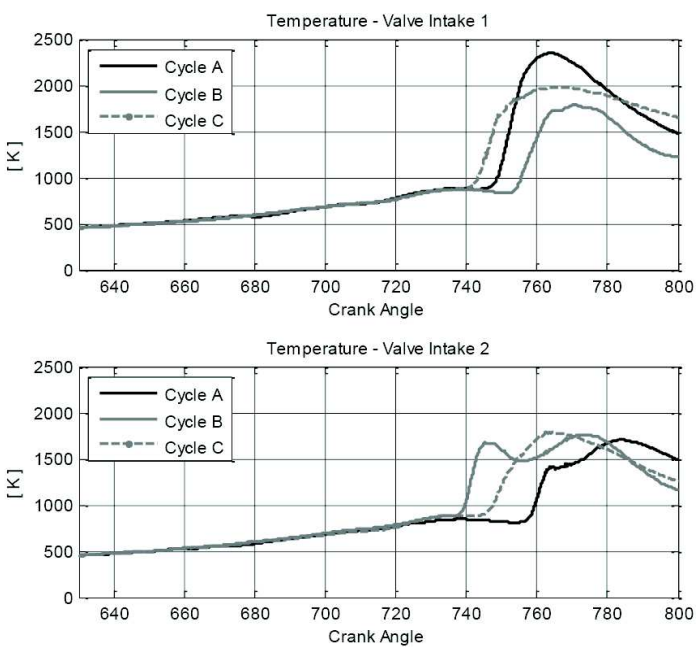

Figure 11a. CCV Analysis: PDFs of Knock Tolerance for the four sectors of the combustion chamber with Experimental SA
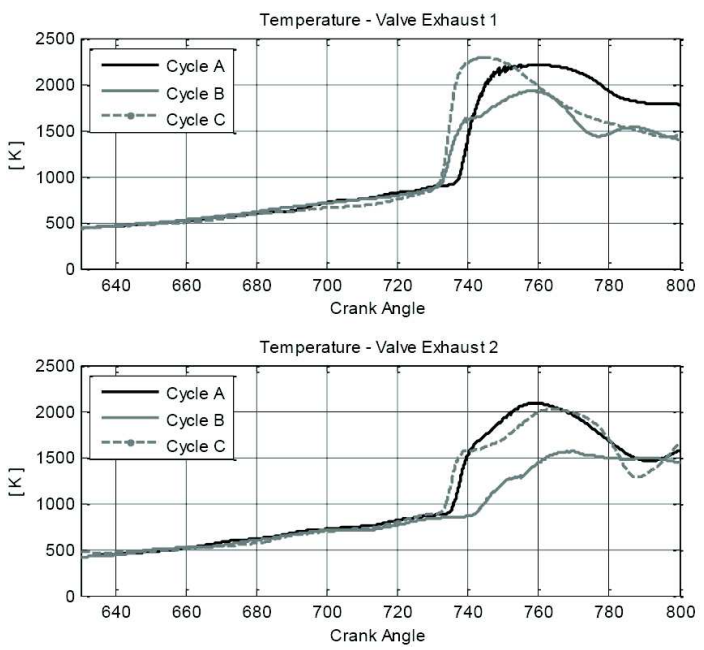
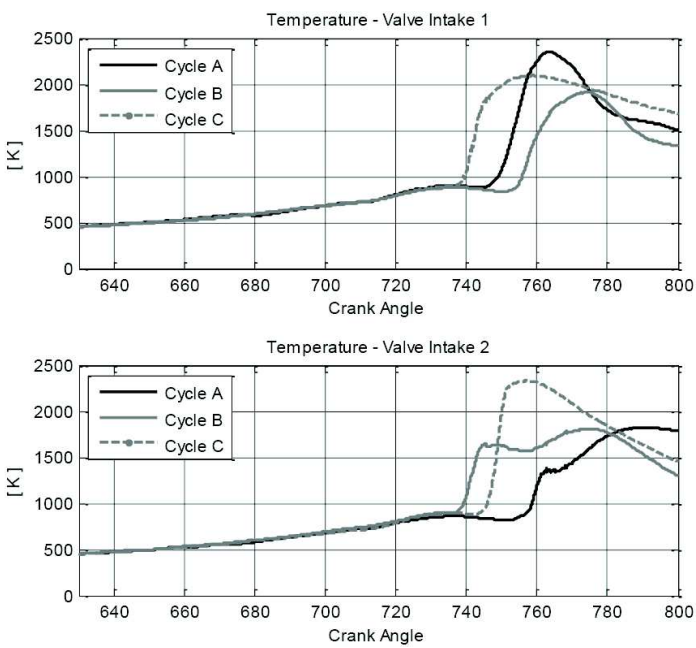

Figure 11b. CCV Analysis : PDFs of Knock Tolerance for the four sectors of the combustion chamber with Moderate Increase

to knock tendency for each of the different sectors of the combustion chamber.

A first set of useful results is the pointwise temperature trace at the previously described four probes located at the outer border of each valve. During most of the combustion development the temperature increase occurring close to the combustion chamber periphery is due only to the compression of the fresh charge by means of the burnt gases. In fact, it is a common approximation to consider the pressure field inside the combustion chamber almost uniform in space during flame propagation (i.e. deflagration); therefore, the unburnt gas temperature variation is the result of the competition between the pressure increase and the wall heat transfer. Towards the final stages of a regular combustion the flame quenching at the walls indicates the time needed for the flame to regularly propagate up to the walls. The passive knock model adopted in this study allows to observe just the effects of a regular propagation and consider separately if the unburnt gas properties and compression history allows that specific regular combustion. The key parameter for this analysis is the CA of flame arrival. From the point of view of local probes at the walls, this corresponds to a sudden increase in temperature measurement. This can be easily identified in the temperature history relieved by each probe by an abrupt increase in the measured value. A threshold limit of $1000 \mathrm{~K}$ is chosen to register the local flame arrival as soon as temperature reaches and exceeds this value.

The temperature traces are reported in Figure 11a, $\underline{b}, \underline{c}$ for the three investigated cycles, and overall results are shown in Table 2. 

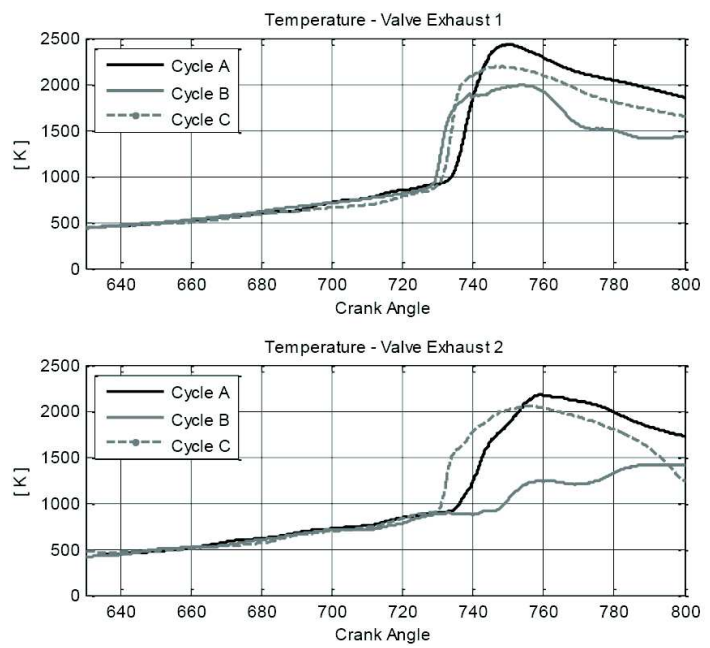
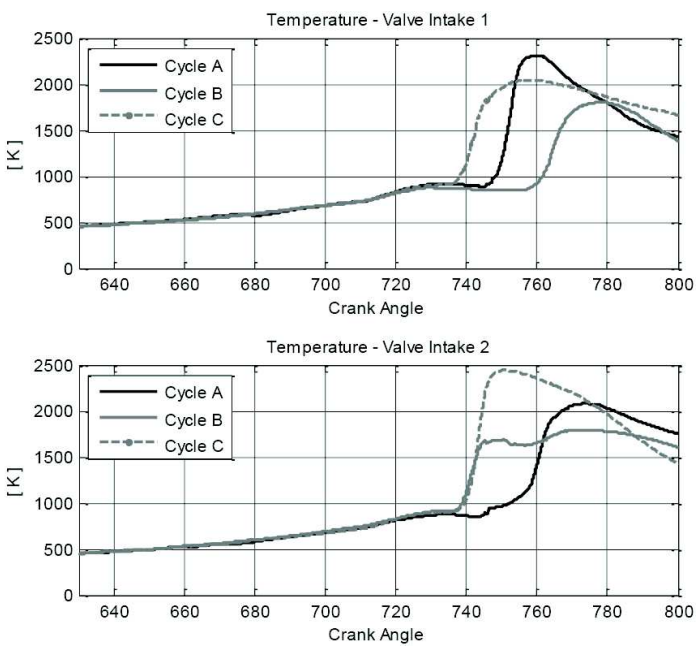

Figure 11c. CCV Analysis: PDFs of Knock Tolerance for the four sectors of the combustion chamber with Moderate Increase $S A$

Table 2. CA of flame quenching at the local wall probes near the valves for the three spark advances investigated

\begin{tabular}{|c|c|c|c|}
\hline \multicolumn{5}{|c|}{ CA for Flame Arrival } \\
\hline $\begin{array}{c}\text { Probe } \\
\text { Location }\end{array}$ & $\begin{array}{c}\text { Experimental } \\
\text { SA } \\
\text { [CA ASOC] }\end{array}$ & $\begin{array}{c}\text { Moderate } \\
\text { Increase SA } \\
\text { [CA ASOC] }\end{array}$ & $\begin{array}{c}\text { High } \\
\text { Increase SA } \\
\text { [CA ASOC] }\end{array}$ \\
\hline $\begin{array}{c}\text { Intake } \\
\text { Valve 1 }\end{array}$ & 43.1 & 45.6 & 49.3 \\
\hline $\begin{array}{c}\text { Intake } \\
\text { Valve 2 }\end{array}$ & 42.8 & 54.9 & 43.6 \\
\hline $\begin{array}{c}\text { Exhaust } \\
\text { Valve 1 }\end{array}$ & 31.2 & 34.5 & 31.6 \\
\hline $\begin{array}{c}\text { Exhaust } \\
\text { Valve 2 }\end{array}$ & 36.1 & 33.0 & 38.8 \\
\hline
\end{tabular}

In order to avoid dependency on the adopted temperature threshold value, the analysis is repeated with a different value of $1300 \mathrm{~K}$. Since no deviation in results is relieved, the presented results can be considered as threshold independent.

The results summarized in Table 2 are averaged over the three investigated LES cycles. The dataset is too limited to introduce a statistical analysis, which is beyond the aims of this study, however it appears clearly that the intake side of the combustion chamber undergoes a very retarded propagation with respect to the exhaust one. This is evident also from a visual analysis of local temperatures in Figure $\underline{11 a, b, \underline{b} . ~ T a b l e ~} 2$ shows also that this characteristic combustion feature is not dependent on the specific SA chosen, and therefore emerges as a main feature of the complex interplay between the residual level of tumble motion, which convects the flame towards the exhaust side, and the spark plug displacement, which is shifted towards the exhaust side. These two effects are thought to mutually enforce a rapid flame propagation on the exhaust side, while the intake charge is subjected to much longer flame propagation times before being completely consumed. This makes the intake side a potentially dangerous location for knock onset.

It is to underline that the very early injection strategy for this operating point leads to an almost homogeneous mixture strength in the unburnt gas, as visible in Figure 12. In addition to this, the injection strategy is specifically designed to deliver an overall high equivalence ratio.

Both these considerations allow to roughly correlate the mass of fuel in the unburnt gases to their volume, and this to be subsequently related to the flame displacement characteristics.
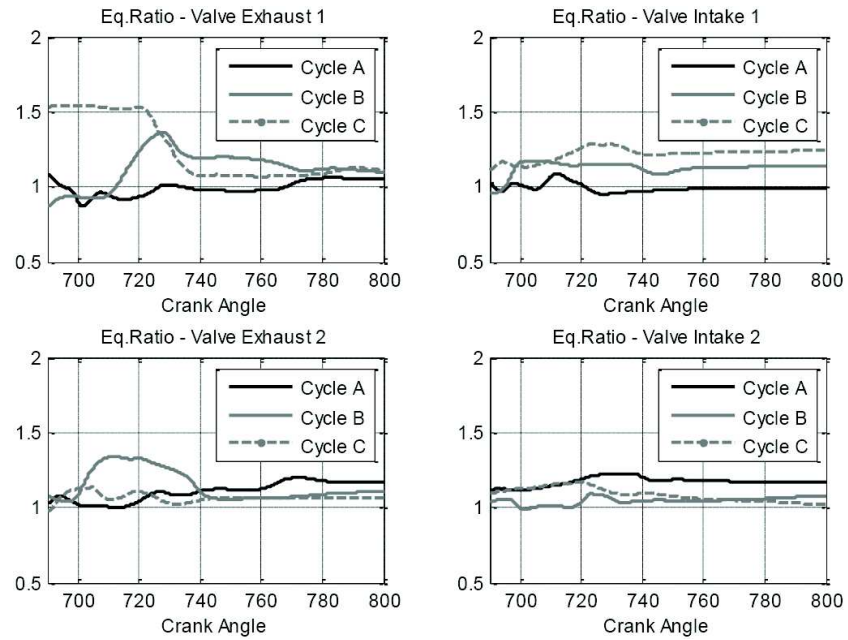

Figure 12. Equivalence ratio relieved in the four sector of the combustion chamber 

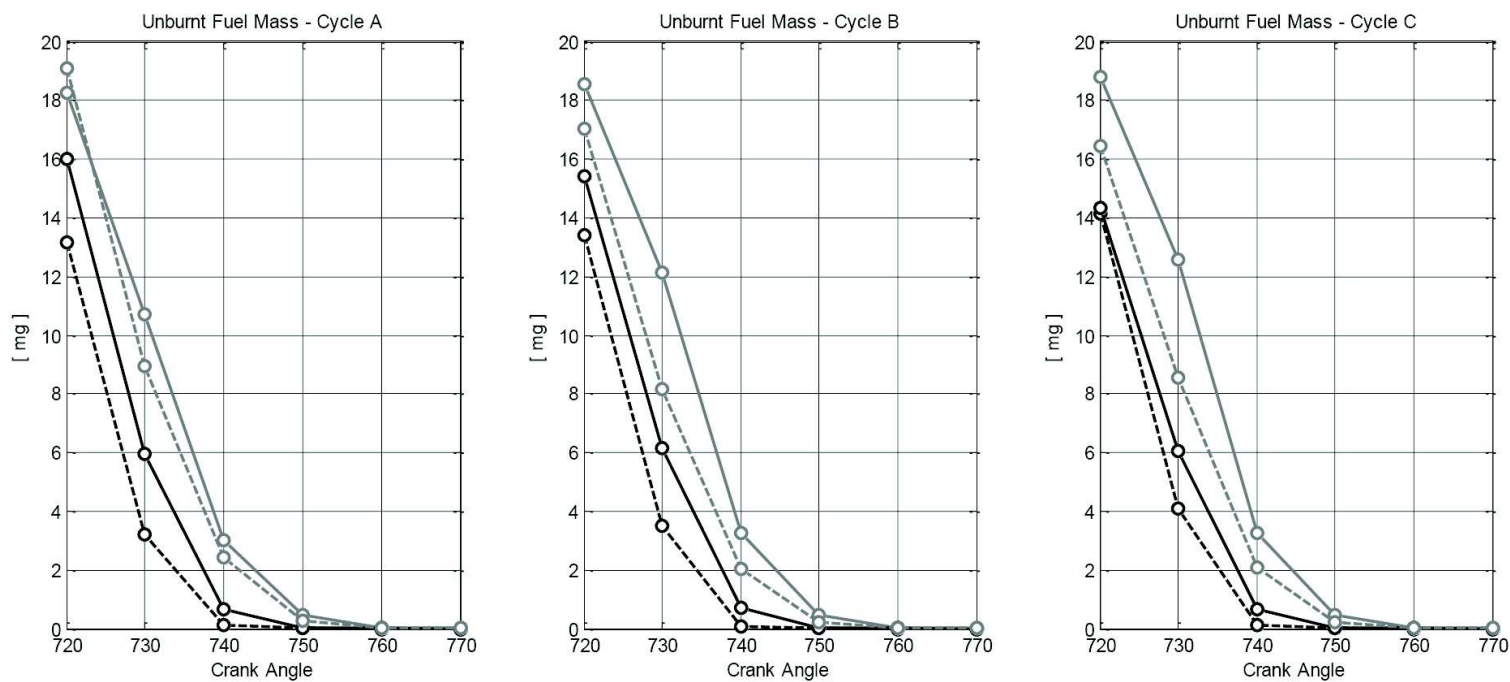

\section{Figure 13. Mass of unburnt fuel in the four sectors of the combustion chamber : intake side (gray solid and dashed lines) and exhaust side (black solid and dashed lines)}

The mass of fuel in the unburnt gas is measured throughout the combustion for the three LES cycles under investigation, as well as for the three SAs. As expected, a reduction in the fuel mass occurs in every sector as combustion proceeds and fuel gets consumed. Within this trend, there is a significant difference between the intake side sectors and the exhaust ones, with the former showing a much larger amount of unburnt fuel. This is clearly visible in Figure 13, which is relative to the experimental SA for the three cycles.

This trait is a consequence of the already depicted flame movement towards the exhaust side of the chamber. As previously discussed, this characteristics is common to every SA investigated, and similar results are observed.

Therefore, the simple detection of a knocking condition from a chemistry viewpoint is not sufficient to evaluate the intensity of the phenomenon, being it also largely dependent on the side of the combustion chamber where it is located and the involved fresh charge mass. As a straightforward example, an autoignition event at the exhaust side is likely to release a very limited amount of energy because of the unburnt fuel shortage due to the faster flame propagation. On the contrary, the same event at the intake side would involve a much larger amount of fuel. The energy liberated by the latter autoignition is expected to be much higher than that of the former.

The mass of fuel reaching autoignition conditions is then related to the mass of unburnt fuel in that specific sector of the combustion chamber. In this way a non-dimensional fraction of fuel showing autoignition conditions is calculated.

Figures 14a, $\underline{b}, \underline{c}$ confirm the aforementioned considerations about the almost null differences in terms of autoignition progress between the two sides of the combustion chamber. Considering the results deriving from Figure 13 it can be finally argued that a heavy knocking event is much more likely to occur in the intake side of the combustion chamber due to the slower combustion development.
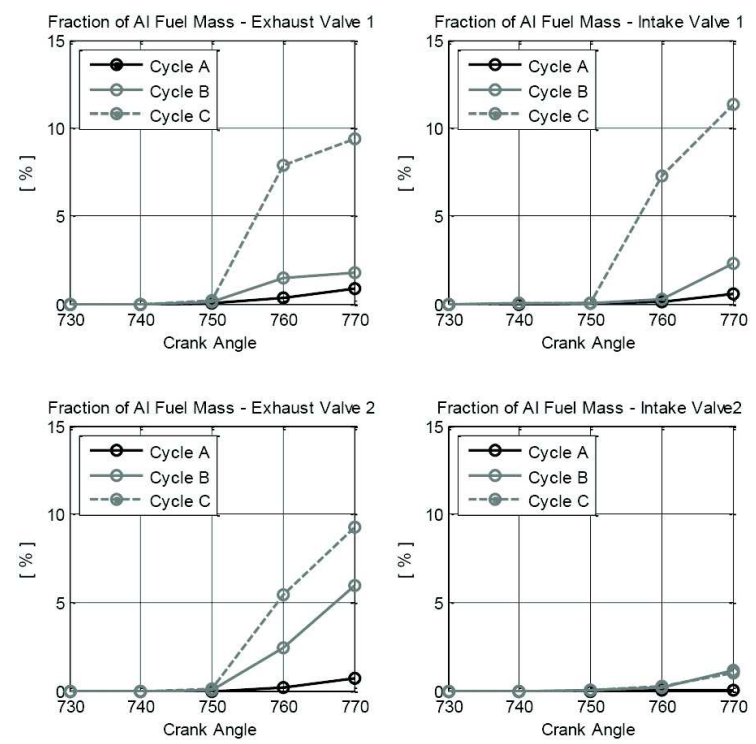

Figure 14a. Fraction of unburnt fuel in autoigniting conditions for experimental $S A$ 

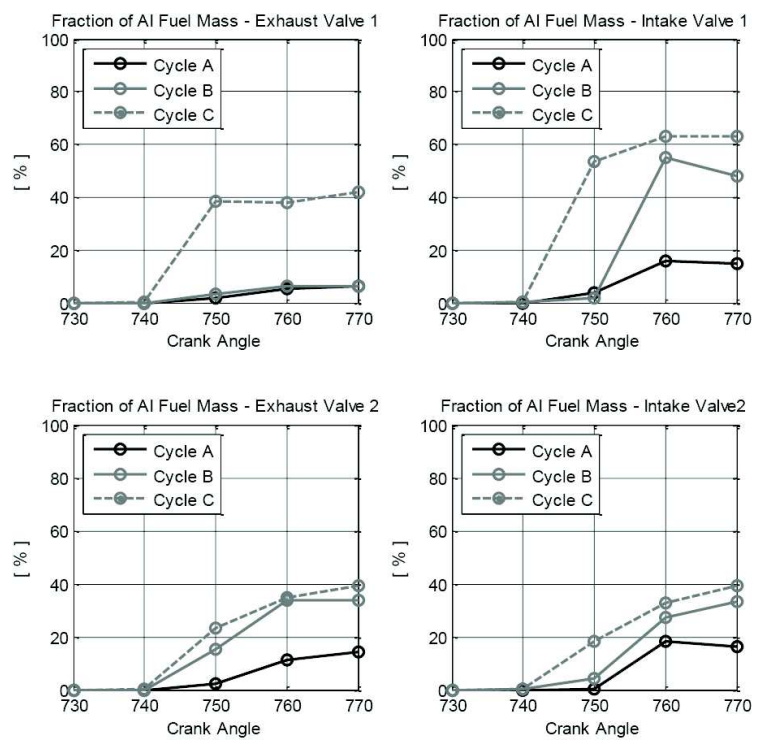

Figure 14b. Fraction of unburnt fuel in autoigniting conditions for moderate increase $S A$
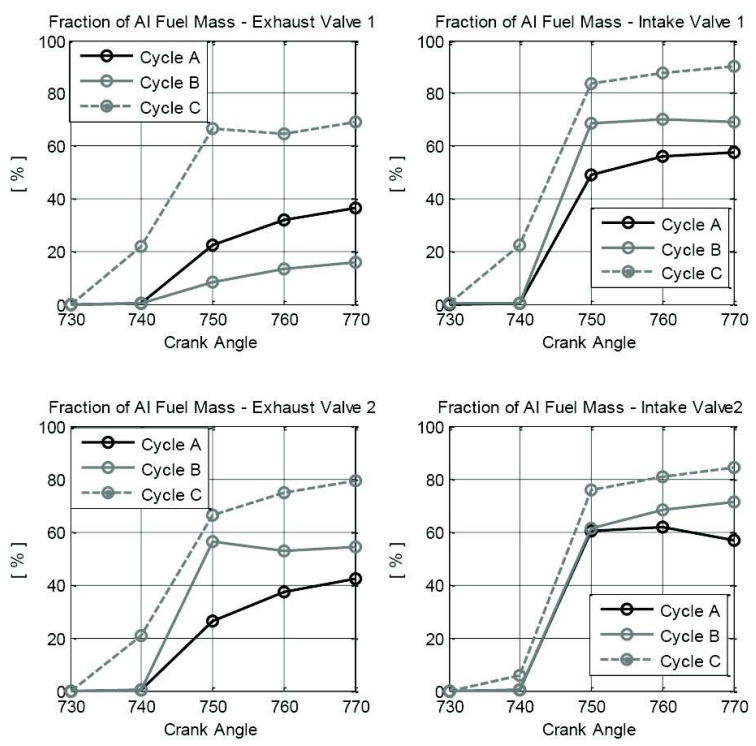

Figure 14c. Fraction of unburnt fuel in autoigniting conditions for high increase $S A$

\section{CONCLUSIONS}

An autoignition model is presented as a stand-alone tool for knocking analysis in CFD simulations of SI engines. In the present study the coupling with the Smagorinsky LES model and ECFM-LES combustion model allows to introduce the intrinsic unsteadiness of LES simulation in knocking analyses. The chemistry for autoignition is modelled by means of look-up tables generated by a dedicated software (DARS Basic) prior to CFD analyses. A first validation is carried out by comparison of three mechanisms/blends for gasoline surrogates. The experimental $\mathrm{SA}$ is evaluated alongside a two-step increase of it, in order to assess the KLSA condition set during the experiments. A sample intermediate cycle is chosen for this validation and all the blends are compared on the same flow realization. The only SA that results in non-knocking conditions is the experimental one, while even for a moderate increase of SA some of the fuel models predicts non-negligible autoigniting conditions. A second part of the study is focused on sensitivity analysis of the proposed knock tool to cyclic dispersion obtained through LES, which proved to be in good agreement with the experimental one. Three relevantly different cycles are tested with a single chemical scheme for gasoline and for the same pattern of increasing SA. Again, for the experimental SA none of the cycles shows any knocking phenomenon, while for moderate and significant increase in SA greatly increased knock tendency is detected.

The methodology assessment for knock characterization presented in this work is intended to be a complete tool for a statistical description of the engine operation and to address engine optimization aiming at both reducing $\mathrm{CCV}$ and increasing overall engine efficiency. The tool proves its predictive capabilities in terms of knock onset timing, and indications are given about favourable locations for abnormal combustion events. Particularly, the developed toolset is characterised by a limited impact on the computational cost of the simulations. In fact, knock-tendency analyses can effectively be restricted to the closed-valve portion of the cycle and several independent cycles can be run in parallel for different spark advances and in-cylinder patterns, in order to build a consistent statistical population of results. For the analyses presented in the paper, each calculation requested about 24 hours of CPU-time on a 24-core system.

\section{REFERENCES}

1. Fontanesi, S., Paltrinieri, S., d'Adamo, A., Duranti, S., "Investigation of boundary condition effects on the analysis of cycle-to-cycle variability of a turbocharged GDI engine", International Conference on LES for Internal Combustion Engine Flows, 2012

2. Rutland, C. J., "Large-eddy simulations for internal combustion engines - A review", doi: 10.1177/1468087411407248.

3. Colin, O., Ducros, F., Veynante, D., Poinsot, T., "A Thickened Flame Model for Large Eddy Simulations of Turbulent Premixed Combustion", Physics of Fluids, Vol. 12 Number 7

4. Kalghatgi, G., "Auto-Ignition Quality of Practical Fuels and Implications for Fuel Requirements of Future SI and HCCI Engines," SAE Technical Paper 2005-01-0239, 2005, doi: 10.4271/2005-01-0239.

5. Pera, C., Knop, V., "Methodology to define gasoline surrogates dedicated to auto-ignition in engines", Fuel 96 (2012) 59-69

6. Lafossas, F., Castagne, M., Dumas, J., and Henriot, S., "Development and Validation of a Knock Model in Spark Ignition Engines Using a CFD code," SAE Technical Paper 2002-01-2701, 2002, doi: 10.4271/2002-01-2701.

7. Douaud, A. and Eyzat, P., "Four-Octane-Number Method for Predicting the Anti-Knock Behavior of Fuels and Engines," SAE Technical Pape 780080, 1978, doi: 10.4271/780080

8. Andrae, J. C. G., Head, R. A., "HCCI Experiments with gasoline surrogate fuels modeled by a semidetailed chemical kinetic model," Combustion and Flame 156 (2009) 842-851

9. Mehl, M., Pitz, W. J., Westbrook, C. K., Curran, H. J., "Kinetic modeling of gasoline surrogate components and mixtures under engine conditions," Proceedings of the Combustion Institute 33:193-200 (2011)

10. Mehl M., Vanhove G., Pitz W.J., Ranzi E., "Combust. Flame 155 (2008) 756-772.

11. DARS Manual Book 2 - Homogeneous Reactor Models 


\section{CONTACT INFORMATION}

Dr. Stefano Fontanesi, Assistant Professor

Department of Engineering "Enzo Ferrari", University of Modena,

Via Vignolese 905 - 41122 Modena (Italy)

Ph. +390592056114

Fax: +390592056126

stefano.fontanesi@unimore.it

Prof. Chistopher J. Rutland

Engine Research Center, Univeristy of Wisconsin-Madison

1500 Engineering Drive - Madison, WI 53706

Ph. +1 608/262-5853

Fax: +1 608/262-6707

rutland@engr.wisc.edu

\section{ACKNOWLEDGMENTS}

This research was funded by Gruppo Motori from University of Modena and Reggio Emilia (Italy) and developed in a joint project with Engine Research Center of University of Wisconsin-Madison (USA). The authors acknowledge and thank Ferrari S.p.A. for experimental data availability and CD-Adapco and DIGANARS for software support.

\section{DEFINITIONS/ABBREVIATIONS}

ASOC - After Start Of Combustion

ATDC - After Top Dead Center

AFTDC - After Firing Top Dead Center

CA - Crank Angle

CCV - Cycle-to-Cycle Variability

DISI - Direct Injection Spark Ignition

ICE - Internal Combustion Engine

KLSA - Knock Limited Spark Advance

LES - Large Eddy Simulation

LLNL - Lawrence Livermore National Laboratory

MFB - Mass Fraction Burnt

NTC - Negative Temperature Coefficient

PRF - Primary Reference Fuel

RANS - Reynolds-Averaged Navier-Stokes

SA - Spark advance

TDC - Top Dead Center

THEO - Toluene / n-Heptane / Ethanol / Isoctane

TRF - Toluene Reference Fuel

WOT - Wide Open Throttle 


\section{APPENDIX}

The flame development is reported by means of the isosurface of 50\% progress variable for the three SA (Experimental SA, $+3 \mathrm{CA}$ and $+6 \mathrm{CA})$. Figure 15 is relative to $+10 \mathrm{CA}$ ATDC.
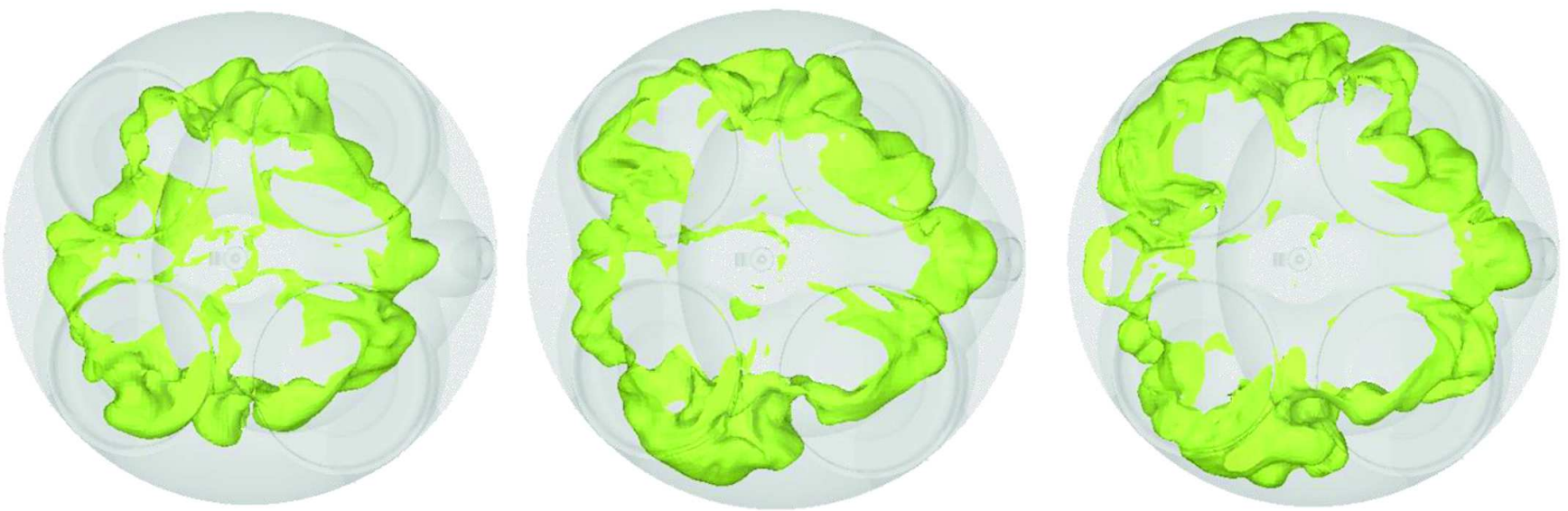

Figure 15. Isosurface at 50\% progress variable at $+10 C A$ ATDC for Experimental SA (left), Moderate Increase SA (middle) and High Increase SA (right)

Figures $16, \underline{17}$ and $\underline{18}$ show the coupling of the CCV resulting from LES simulation with knock prediction operated by the autoignition tool. The regular combustion development is represented by the isosurface at 50\% of progress variable (yellow isosurface), while areas of occurred autoignition are represented by red isosurface. Figures $16, \underline{17}$ and $\underline{18}$ are relative to $+30 \mathrm{CA}$ ATDC (about 90\% MFB).
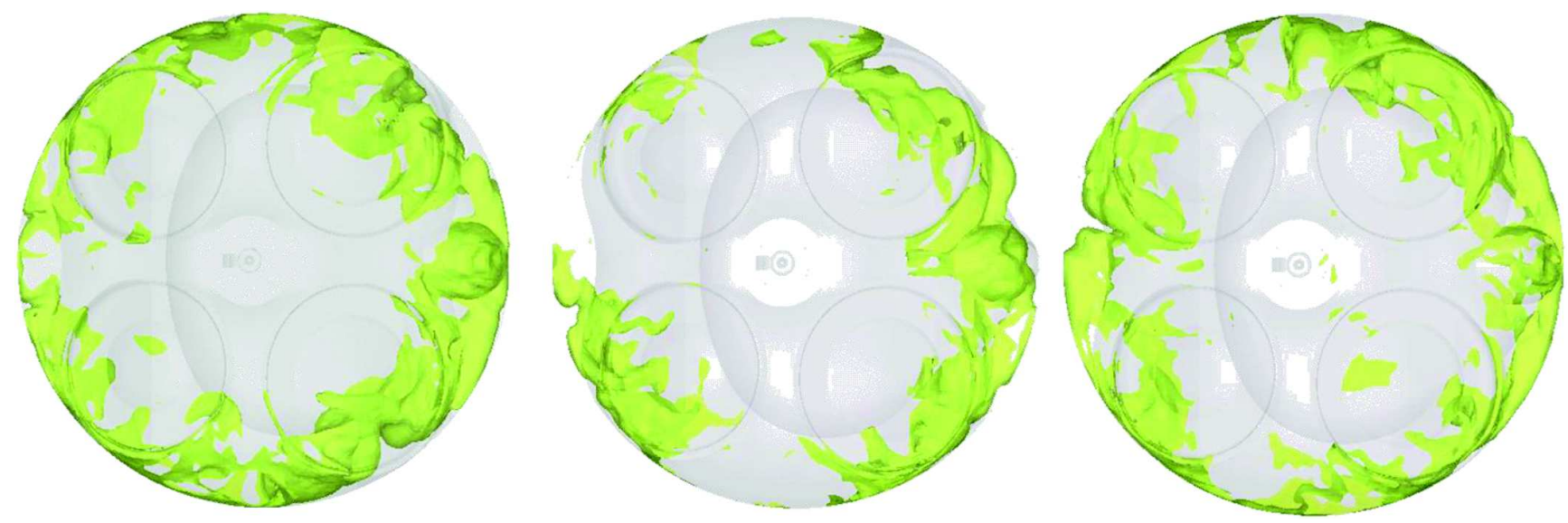

Figure 16. Combustion development and autoignition areas at +30CA ATDC for Experimental SA: Cycle A (left), Cycle B (middle), Cycle C (right). 

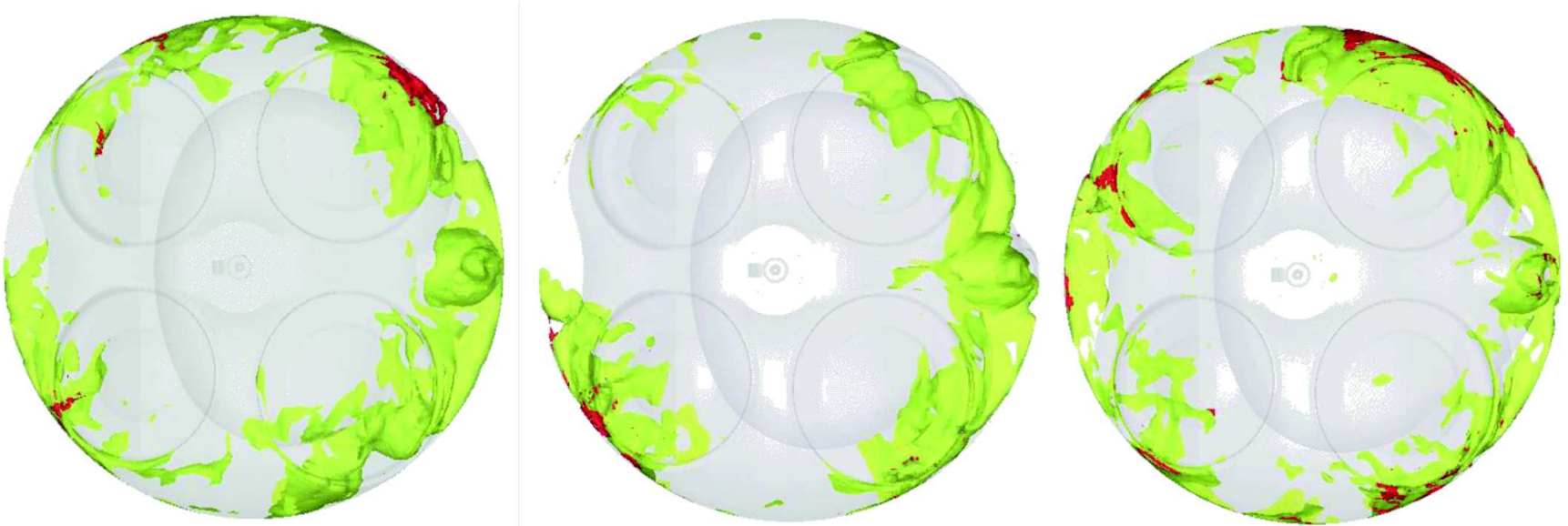

Figure 17. Combustion development and autoignition areas at +30CA ATDC for Moderate Increase SA: Cycle A (left), Cycle B (middle), Cycle C (right).
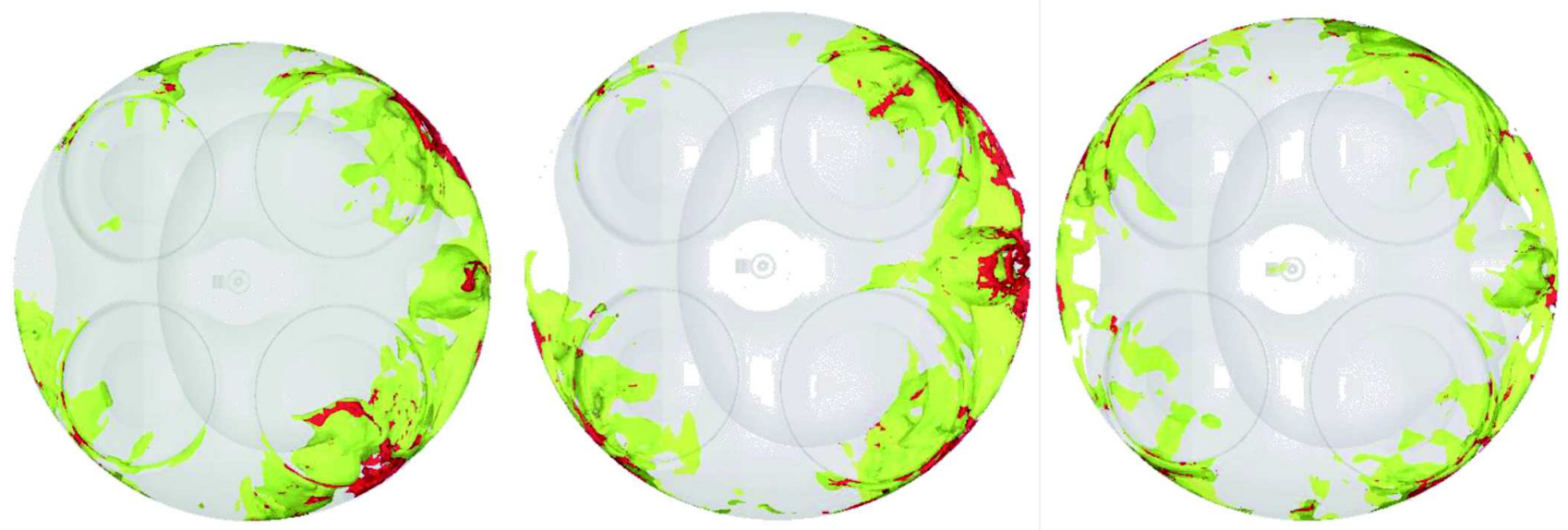

Figure 18. Combustion development and autoignition areas at +30CA ATDC for High Increase SA: Cycle A (left), Cycle B (middle), Cycle C (right). 